Article

\title{
Additive SMILES-Based Carcinogenicity Models: Probabilistic Principles in the Search for Robust Predictions
}

\author{
Andrey A. Toropov ${ }^{1,2, *}$, Alla P. Toropova ${ }^{1,2}$ and Emilio Benfenati ${ }^{2}$ \\ 1 Institute of Geology and Geophysics, 100041, Khodzhibaev St. 49, Tashkent, Uzbekistan; \\ E-Mail: altoropova@mail.ru (A.P.T.) \\ 2 Istituto di Ricerche Farmacologiche Mario Negri, 20156, Via La Masa 19, Milano, Italy; \\ E-Mail: benfenati@marionegri.it (E.B.) \\ * Author to whom correspondence should be addressed; E-Mail: aatoropov@yahoo.com (A.A.T.); \\ Tel. +390239014595; Fax: +390239014735
}

Received: 14 May 2009; in revised form: 23 June 2009 / Accepted: 2 July 2009 /

Published: 8 July 2009

\begin{abstract}
Optimal descriptors calculated with the simplified molecular input line entry system (SMILES) have been utilized in modeling of carcinogenicity as continuous values $\left(\log \mathrm{TD}_{50}\right)$. These descriptors can be calculated using correlation weights of SMILES attributes calculated by the Monte Carlo method. A considerable subset of these attributes includes rare attributes. The use of these rare attributes can lead to overtraining. One can avoid the influence of the rare attributes if their correlation weights are fixed to zero. A function, limS, has been defined to identify rare attributes. The limS defines the minimum number of occurrences in the set of structures of the training (subtraining) set, to accept attributes as usable. If an attribute is present less than limS, it is considered "rare", and thus not used. Two systems of building up models were examined: 1. classic training-test system; 2. balance of correlations for the subtraining and calibration sets (together, they are the original training set: the function of the calibration set is imitation of a preliminary test set). Three random splits into subtraining, calibration, and test sets were analysed. Comparison of abovementioned systems has shown that balance of correlations gives more robust prediction of the carcinogenicity for all three splits (split $1: \mathrm{r}_{\text {test }}{ }^{2}=0.7514, \mathrm{~s}_{\text {test }}=0.684$; split 2: $\mathrm{r}_{\text {test }}^{2}=0.7998, \mathrm{~s}_{\text {test }}=0.600$; split 3: $\left.\mathrm{r}_{\text {test }}^{2}=0.7192, \mathrm{~s}_{\text {test }}=0.728\right)$.
\end{abstract}


Keywords: QSAR; SMILES; optimal descriptor; carcinogenicity; balance of correlations; applicability domain

\section{Introduction}

Carcinogenicity is an important endpoint from a toxicological point of view and quantitative structure - activity relationships (QSAR) are a tool for modeling this endpoint [1-3]. Usually, the QSAR analysis is based on molecular descriptors, calculated from molecular graphs [3,4]. However, the simplified molecular input line entry system (SMILES) [5-7] has become a prospective alternative to molecular graphs in QSAR analysis [8-11], owing to an expansion of the databases available via the Internet with molecular structures given in SMILES notation $[15,16]$. The present study aimed to estimate the ability of the SMILES-based optimal descriptors to be a tool for QSAR analysis of carcinogenicity of non-congeneric chemicals.

\section{Materials and Methods}

Carcinogenicity data: Experimental values for carcinogenicity were taken from publicly available data sources and further checked for chemical structures [17]. Carcinogenicity is expressed as the potency dose that induces cancer in rats $\left(\mathrm{TD}_{50}\right.$, in $\mathrm{mg} / \mathrm{kg}$ body weight). These values have been converted into $\mathrm{mmol} / \mathrm{kg}$ body weight. The $-\log \left(\mathrm{TD}_{50}\right)$ was examined as endpoint for the modelling. Initially, 401 chemicals have been extracted from [17]. These compounds were selected as substances with numerical data on the carcinogenicity available from [17].

However, this set (401 compounds) contains eight outliers (Table 1): for these compounds the difference between experimental and calculated (by our approach) value of -logTD50 is more than the double the standard error (2s). Probably the high symmetry and the presence of the $N$-nitroso group can lead to the unusual behaviour of these substances. These compounds were removed. Thus, 393 compounds were examined in this study. SMILES notations which were used in this study have been taken from [18].

We randomly split these 393 chemicals three times into training $(n=165)$, calibration $(n=167)$ and test $(n=61)$ sets. The range of $-\log \left(\mathrm{TD}_{50}\right)$ values for these sets is about from -2 to 5 logarithmic units. Below, these splits are denoted the Split1, Split2, and Split3 (The Supplementary Materials contain lists of these splits).

The modification of the descriptor that was used for modeling bee toxicity [10] is the tool for QSAR analysis of the carcinogenicity. This descriptor is calculated as follows:

$$
\mathrm{DCW}(\operatorname{limS})=\mathrm{CW}(\mathrm{dC})+\boldsymbol{\Sigma} \mathrm{CW}\left({ }^{1} \mathrm{SA}_{\mathrm{k}}\right)+\boldsymbol{\Sigma} \mathrm{CW}\left({ }^{2} \mathrm{SA}_{\mathrm{k}}\right)+\boldsymbol{\Sigma} \mathrm{CW}\left({ }^{3} \mathrm{SA}_{\mathrm{k}}\right)
$$

where ${ }^{1} \mathrm{SA}_{\mathrm{k}},{ }^{2} \mathrm{SA}_{\mathrm{k}},{ }^{3} \mathrm{SA}_{\mathrm{k}}$ are SMILES attributes. ${ }^{1} \mathrm{SA}_{\mathrm{k}},{ }^{2} \mathrm{SA}_{\mathrm{k}}$, and ${ }^{3} \mathrm{SA}$ contain one, two, and three SMILES elements, respectively. The SMILES element can be one (e.g., 'C', 'c', 'N', 'S', etc.), two (e.g., ' $\mathrm{Cl}$ ', 'Br', etc.), three (' $\mathrm{C}=\mathrm{O}$ '), and four symbols ('[O-]'). The order of elements in depiction of the ${ }^{2} \mathrm{SA}_{\mathrm{k}}$ or ${ }^{3} \mathrm{SA}_{\mathrm{k}}$ is defined by the ASCII characters. In other words only one version of AB-sequence 
or $\mathrm{ABC}$-sequence is possible in the list of the SMILES-attributes (not $\mathrm{AB}$ together with $\mathrm{BA}$, or $\mathrm{ABC}$ together with $\mathrm{CBA}$ ).

Table 1. The list of outliers of the QSAR models calculated with SMILES-based optimal descriptors.

\begin{tabular}{|c|c|c|c|}
\hline Number & Structure & CAS & Chemical name \\
\hline 1 & $\mathrm{O}^{-} \mathrm{H}_{3} \mathrm{C}$ & $606-20-2$ & 2,6-Dinitrotoluene \\
\hline 2 & & $57497-34-4$ & Z-Methyl-O,N,N-azoxyethane \\
\hline 3 & & $17608-59-2$ & $N$-Nitrosoephedrine \\
\hline 4 & & $15973-99-6$ & $\operatorname{Di}(N$-nitroso)-perhydropyrimidine \\
\hline 5 & & $61034-40-0$ & $\begin{array}{l}\text { 1-Nitroso-4-benzoyl-3,5- } \\
\text { dimethylpiperazine }\end{array}$ \\
\hline 6 & & $99-80-9$ & $N, 4$-Dinitrosomethylaniline \\
\hline 7 & 0 & $55557-00-1$ & $N, N$-Dinitrosohomopiperazine \\
\hline 8 & & $86-30-6$ & $\mathrm{~N}$-Nitrosodiphenylamine \\
\hline
\end{tabular}


The $\mathrm{dC}$ is the difference of the number of ' $\mathrm{C}$ ' (capital letter) in the given SMILES notation minus the number of 'c' (lowercase letter) in the given SMILES notation. For example, this global SMILES attribute is denoted as ' $! 001$ ', if $\mathrm{dC}=\mathrm{N}\left({ }^{\prime} \mathrm{c} \mathrm{c}^{\prime}\right)-\mathrm{N}\left({ }^{\prime} \mathrm{C}\right.$ ') $=1$, and as ' !- 02 ' if the $\mathrm{dC}=-2$. The $\mathrm{CW}(\mathrm{dC})$ is the correlation weight of the $\mathrm{dC}$. The symbol "C" (capital letter) is the representation of a carbon atom in the $\mathrm{sp}^{3}$ configuration. The symbol " $\mathrm{c}$ " (lowercase letter) is the representation of a carbon atom in $\mathrm{sp}^{2}$ configuration. Thus, the $\mathrm{dC}$ is a measure of presence of rigid and flexible fragments in molecular architecture. The examined substances contain chlorine that gives an additional ' $\mathrm{C}$ '. The chlorine is not rigid fragment in molecular system and we have calculated the $\mathrm{dC}$ taking into account the ' $\mathrm{C}$ ' from chlorine atoms. Table 2 contains an example of the representation of SMILES by the set of SMILES attributes.

Table 2. Example of definition of SMILES attributes (unused positions are indicated by dots).

\begin{tabular}{|l|l|l|l|l|l|}
\hline${ }^{1} \mathbf{S}_{\mathbf{k}}$ & $\mathbf{C W}\left({ }^{1} \mathbf{S}_{\mathbf{k}}\right)$ & ${ }^{2} \mathbf{S}_{\mathbf{k}}$ & $\mathbf{C W}\left({ }^{2} \mathbf{S}_{\mathbf{k}}\right)$ & $\mathbf{d C}$ & $\mathbf{C W}(\mathbf{d C})$ \\
\hline $\mathrm{C} \ldots \ldots \ldots . .$. & -0.0156855 & & & & \\
$\mathrm{O}=\mathrm{C} \ldots \ldots \ldots$. & -2.8475657 & $\mathrm{O}=\mathrm{C} . \mathrm{C} \ldots \ldots$. & 0.0 & $!-02 \ldots \ldots \ldots$ & 1.2190257 \\
\hline
\end{tabular}

SMILES $=" \mathrm{CC}=\mathrm{O} " ; \mathrm{CAS}=75-07-0 ; \mathrm{DCW}=-1.6442255$.

The $\mathrm{CW}(\mathrm{dC}), \mathrm{CW}\left({ }^{1} \mathrm{SA}_{\mathrm{k}}\right), \mathrm{CW}\left({ }^{2} \mathrm{SA}_{\mathrm{k}}\right)$, and $\mathrm{CW}\left({ }^{3} \mathrm{SA}_{\mathrm{k}}\right)$ are correlation weights of the above SMILES attributes. By means of the Monte Carlo method one can calculate numerical data for these weights which give maximal value of determination coefficient (square of the correlation coefficient, $\mathrm{r}^{2}$ ) for the training set. However, most probably overtraining will result, i.e., an excellent model on the training set will be accompanied by a poor model for the test set. In order to avoid overtraining one can use the correlation balance [11], i.e., split the available chemicals into three sets: subtraining, calibration, and external test set. This approach gave reasonable result for the case of toxicity of 61 compounds [11], however for carcinogenicity of 393 compounds it is not enough. The use of the correlation balance and blocking of rare SMILES attributes [10] can improve the model. The blocking of rare attributes can be done by the scheme: if the number of SMILES from the training (subtraining) set which contain the SMILES attribute SA* is less than the $\operatorname{limS}$, the correlation weight of the SA* should be fixed equal to zero, $\mathrm{CW}\left(\mathrm{SA}^{*}\right)=0$.

Without rare attributes the model becomes better for the external test set. However, if limS is too large, the predictive potential of the model decreases, because the low number of active SMILES attribute cannot provide a high quality model. Thus, the central point of the system of modeling is the selection of the most efficient limS. The general scheme of the construction of optimal SMILES-based descriptors by the correlation balance method is represented in Figure 1.

This system can be denoted as a [Subtraining-Calibration-Test] system. The model can be satisfactory if the $\mathrm{N}_{111}$, i.e., the number of active (not blocked) attributes which are present in subtraining, calibration, and test sets, is as large as possible. The more traditional, "classic" approach is the construction of the model using united training set to predict the endpoint for an external test set. This system can be denoted as [Training-Test] system. This model can be satisfactory if the $\mathrm{N}_{101}$, i.e., the number of active attributes which are present in both the training and test set is as large as possible.

The correlation weights were calculated by the Monte Carlo method Optimization. The [TrainingTest] system is based on correlation weights which provide maximum of the correlation coefficient 
between $\mathrm{DCW}(\operatorname{limS})$ and $\log \left(\mathrm{TD}_{50}\right)$ for the training set. The [Subtraining-Calibration-Test] system is based on correlation weights which provide the maximum of a target function (TF) calculated as follows [11-14]:

$$
\mathrm{TF}=\mathrm{D}(\text { subtraining })+\mathrm{D}(\text { calibration })-\mathrm{ABS}[\mathrm{D}(\text { subtraining })+\mathrm{D}(\text { calibration })] \cdot 0.1
$$

where $\mathrm{D}$ (subtraining) and $\mathrm{D}$ (calibration) are determination coefficients between $\mathrm{DCW}(\mathrm{limS})$ and $\log \left(\mathrm{TD}_{50}\right)$ for subtraining and calibration sets, respectively. Thus the optimization for the above system has been carried out by the same algorithm [11], but with different target functions.

Figure 1. General scheme of construction of the optimal SMILES-based descriptors by means of the correlation balance method.

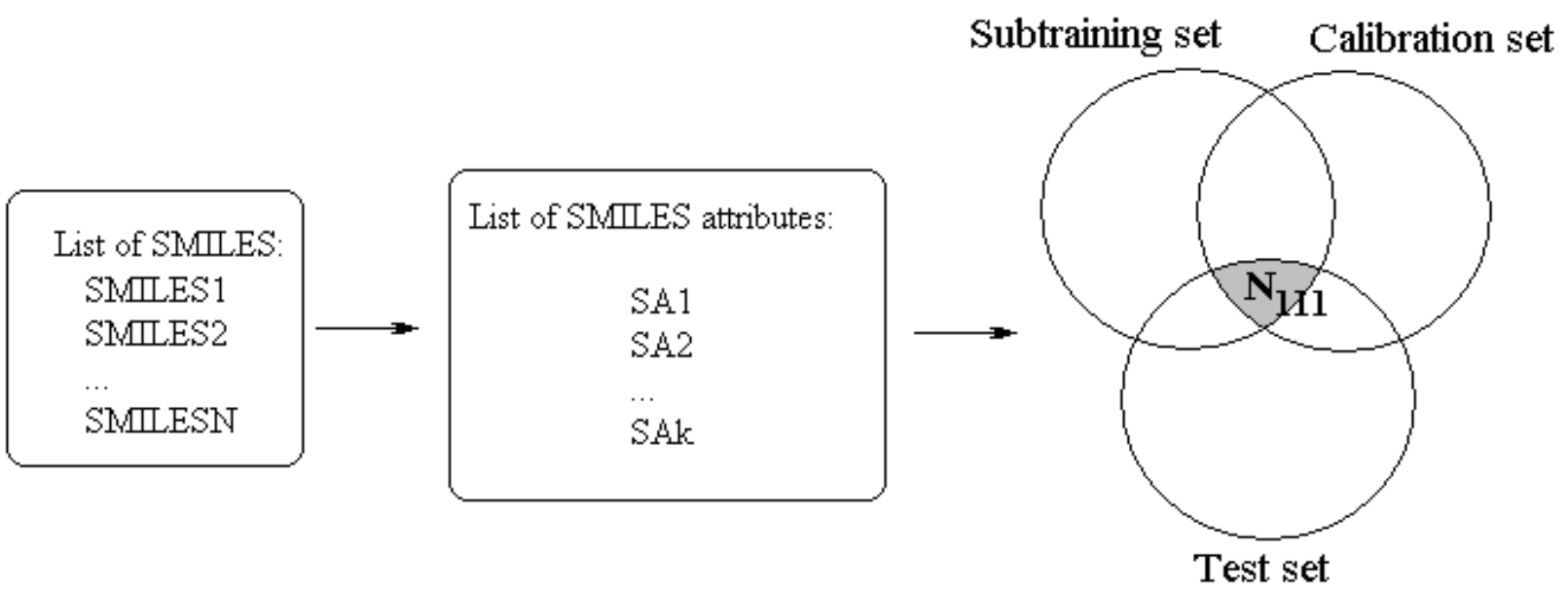

Phase 1. The definition of general list of the SMILES attributes $(\operatorname{limS}=0)$. The $\mathrm{N}_{111}$ is the number of the attributes which are present in the subtraining, in calibration, and in test set. If $\operatorname{limS}=0$ the $\mathrm{N}_{111}$ is relatively low.

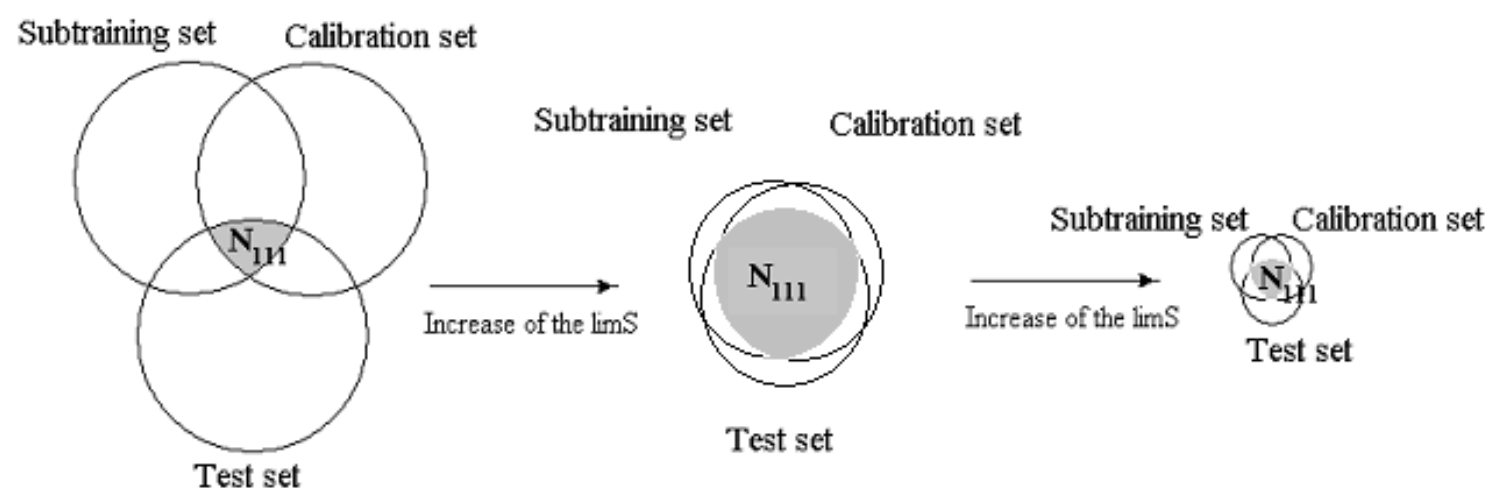

Subtraining set: model is ideal Calibration set: model is ideal Test set: model is poor
Subtraining set: model is reasonable Calibration set: model is reasonable Test set: model is reasonable
Subtraining set: model is poor Calibration set: model is poor Test set: model is poor

$$
(\operatorname{limS}=0)
$$

$$
\text { (limS }{ }^{*} \text { is most productive) }
$$

Phase 2. The definition of the most productive limS value: $0<\operatorname{limS} *<\infty$; this value gives maximum of the $\mathrm{N}_{111}$, i.e., number of the SMILES attributes which are present in the subtraining, in calibration, and in test set. 
For each attribute $\mathrm{SA}, \mathrm{CW}(\mathrm{SA})$ is determined initially by setting the start values of all CWs to $1 \pm$ $0.01 *$ random. The random is the generator of random value of range $(0,1)$. The regular order of number of attributes (i.e., 1, 2, 3, 4, 5,..) is replaced by a random sequence (e.g., 3, 1, 5, 2, 4,...). A starting value of target function (TF1) is calculated. In a generated random sequence, each attribute correlation weight $\mathrm{CWi}$ was modified with the algorithm:

1. DCWi: $=0.5^{*} \mathrm{CWi}$; Eps: $=0.1 * \mathrm{DCW}$;

2. Calculation of TF1; CWi: $=\mathrm{CWi}+\mathrm{DCWi}$;

3. Calculation of TF2, after modify CWi;

4. If TF2 > TF1 then TF1:=TF2; go to 2

5. $\mathrm{CWi}:=\mathrm{CWi}-\mathrm{DCW}$;

6. DCWi:= $-0.5 * \mathrm{DCW}$;

7. If absolute value (DCWi) $>$ Eps then go to 2 .

Then, steps of 1-7 are carried out for all CWs (the epoch of the optimization). By computational experiment the optimal number of the epochs has been established (Table 3). This number is 10 (Figure 2).

Figure 2. Results of computational experiments, which were used to establish of the preferable number of epochs of the Monte Carlo optimization (Nepoch). Triangles indicate curves for the test sets. Black circles denote the sub training set. White circles denote the calibration set.

[ Subtraining (*)-Calibration ( 0 )-Test $(*)$ ] system

[ Training (*)-Test $(4)$ ] system
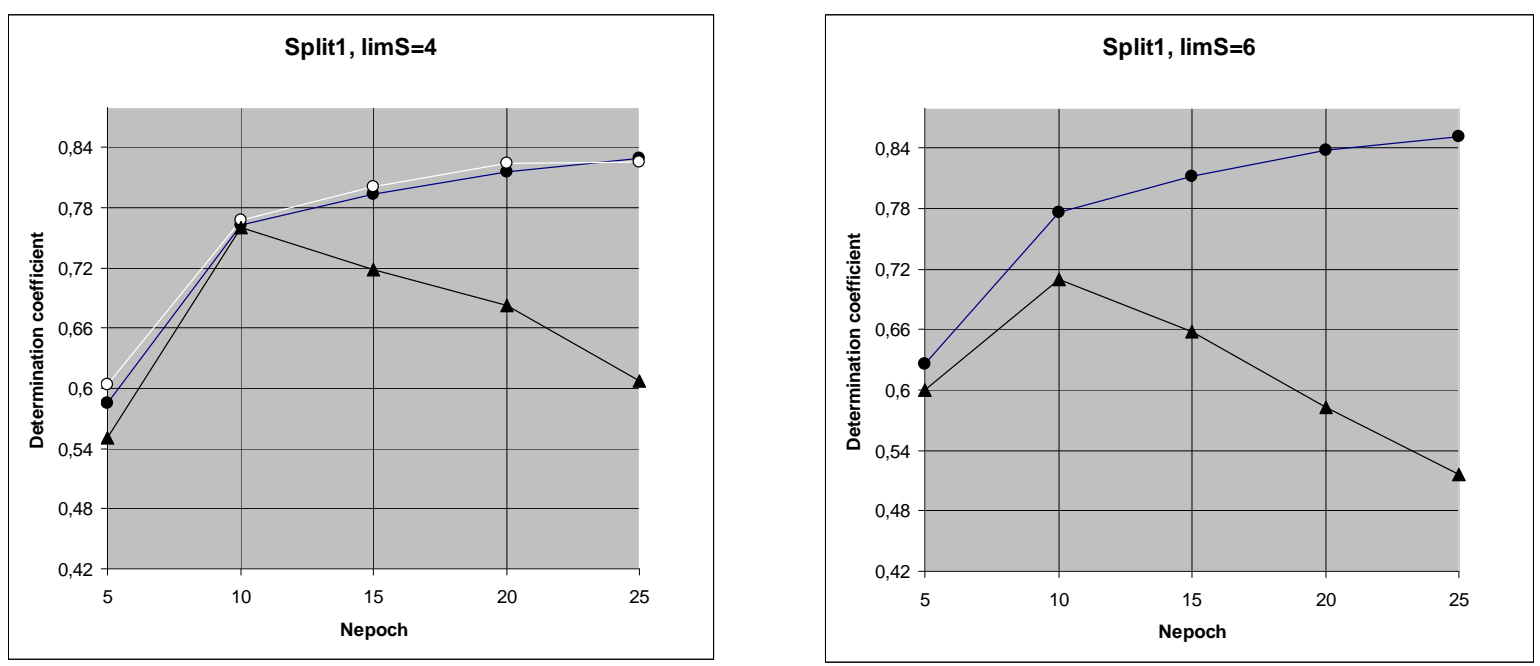
Figure 2. Cont.
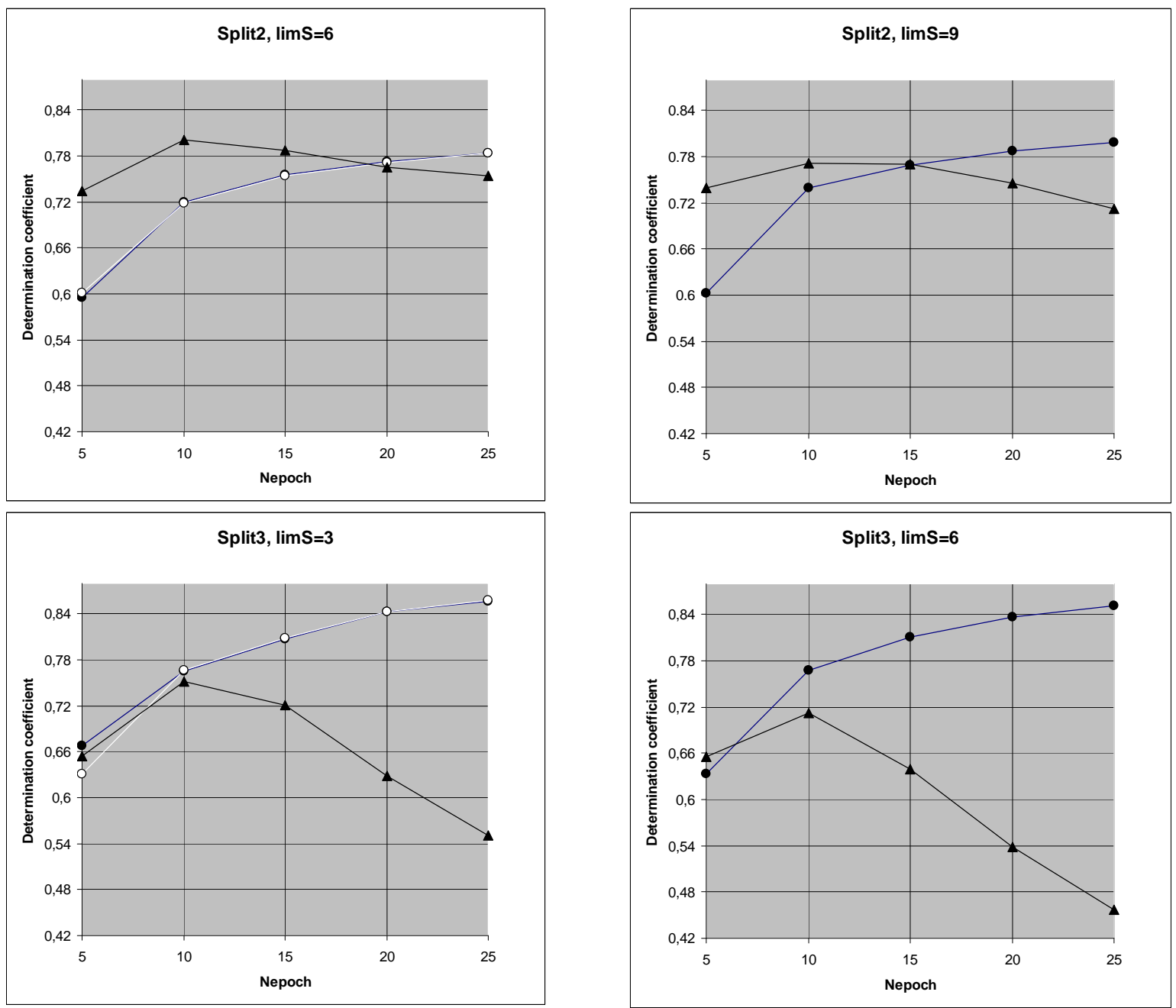

Table 3. Results of computational experiments to establish of number of epochs of the Monte Carlo optimization, Nepoch.

\begin{tabular}{|c|c|c|c|}
\hline \multicolumn{4}{|c|}{ [Subtraining-Calibration-Test] system } \\
\hline Nepoch & $\mathbf{r}_{\text {subtraining }}^{2}$ & $\mathbf{r}_{\text {calibration }}^{2}$ & $\mathbf{r}_{\text {test }}^{2}$ \\
\hline \multicolumn{4}{|c|}{ Split-1 } \\
\hline 5 & 0.5850 & 0.6043 & 0.5513 \\
\hline 10 & 0.7629 & 0.7675 & 0.7601 \\
\hline 15 & 0.7939 & 0.8006 & 0.7187 \\
\hline 20 & 0.8154 & 0.8243 & 0.6827 \\
\hline 25 & 0.8300 & 0.8262 & 0.6076 \\
\hline \multicolumn{4}{|c|}{ Split-2 } \\
\hline 5 & 0.5947 & 0.6017 & 0.7347 \\
\hline 10 & 0.7195 & 0.7190 & 0.8011 \\
\hline 15 & 0.7551 & 0.7538 & 0.7870 \\
\hline 20 & 0.7732 & 0.7719 & 0.7659 \\
\hline 25 & 0.7839 & 0.7834 & 0.7538 \\
\hline
\end{tabular}


Table 3. Cont.

\begin{tabular}{|c|c|c|c|}
\hline \multicolumn{4}{|c|}{ Split-3 } \\
\hline 5 & 0.6673 & 0.6303 & 0.6548 \\
\hline 10 & 0.7656 & 0.7669 & 0.7519 \\
\hline 15 & 0.8077 & 0.8080 & 0.7205 \\
\hline 20 & 0.8436 & 0.8428 & 0.6288 \\
\hline 25 & 0.8562 & 0.8581 & 0.5503 \\
\hline \multicolumn{4}{|c|}{$\begin{array}{c}\text { [Training-Test] system } \\
\text { Split-1 }\end{array}$} \\
\hline 5 & 0.6255 & & 0.6003 \\
\hline 10 & 0.7761 & & 0.7098 \\
\hline 15 & 0.8124 & & 0.6579 \\
\hline 20 & 0.8386 & & 0.5826 \\
\hline 25 & 0.8521 & & 0.5158 \\
\hline \multicolumn{4}{|c|}{ Split-2 } \\
\hline 5 & 0.6028 & & 0.7397 \\
\hline 10 & 0.7396 & & 0.7719 \\
\hline 15 & 0.7687 & & 0.7705 \\
\hline 20 & 0.7872 & & 0.7452 \\
\hline 25 & 0.7985 & & 0.7123 \\
\hline \multicolumn{4}{|c|}{ Split-3 } \\
\hline 5 & 0.6328 & & 0.6559 \\
\hline 10 & 0.7682 & & 0.7127 \\
\hline 15 & 0.8109 & & 0.6397 \\
\hline 20 & 0.8368 & & 0.5378 \\
\hline 25 & 0.8519 & & 0.4573 \\
\hline
\end{tabular}

\section{Results}

Computational experiments (Figure 3, Table 4) have shown that [Subtraining-Calibration-Test] system gives preferable results in comparison with the [Training-Test] system for all three splits. Thus the correlation balance (i.e., [Subtraining-Calibration-Test] system) improves QSAR model of $\log (\mathrm{TD} 50)$. It is the second successful experiment using the correlation balance for the QSAR analyses [11].

Table 4. Average statistical characteristics of the QSAR model of carcinogenicity $\left(\log \mathrm{TD}_{50}\right)$ for three splits into the subtraining, calibration, and test sets with the limS values of $0-10$. For the best models three attempts of the Monte Carlo optimization together with average values are presented, for other models only average values are shown.

\section{SPLIT1}

\begin{tabular}{|c|c|c|c|}
\hline $\begin{array}{c}\text { Subtraining set, } \\
\mathbf{n}=\mathbf{1 6 5}\end{array}$ & $\begin{array}{c}\text { Calibration set, } \\
\mathrm{n}=167\end{array}$ & $\begin{array}{c}\text { Test set, } \\
\mathbf{n}=\mathbf{6 1}\end{array}$ & $\begin{array}{c}\mathrm{SA}_{\mathbf{k}} \\
\text { distribution }\end{array}$ \\
\hline
\end{tabular}


Table 4. Cont.

[Subtraining-Calibration-Test] system

\begin{tabular}{|c|c|c|c|c|c|c|c|c|c|c|c|c|}
\hline $\operatorname{limS}$ & Nact & $\mathrm{R}^{2}$ & $\mathrm{~S}$ & $\mathrm{~F}$ & $\mathrm{R}^{2}$ & $\mathrm{~s}$ & $\mathrm{~F}$ & $\mathrm{R}^{2}$ & $\mathrm{~s}$ & $\mathrm{~F}$ & $\mathrm{~W} \%$ & $\mathrm{~N}_{111}$ \\
\hline 0 & 797 & 0.8731 & 0.500 & 1125 & 0.8805 & 0.619 & 1217 & 0.5769 & 0.893 & 81 & 42 & 333 \\
\hline 1 & 622 & 0.8807 & 0.485 & 1203 & 0.8821 & 0.621 & 1235 & 0.5319 & 0.942 & 67 & 50 & 314 \\
\hline 2 & 407 & 0.8275 & 0.583 & 783 & 0.8268 & 0.703 & 789 & 0.6305 & 0.832 & 101 & 70 & 285 \\
\hline 3 & 321 & 0.7801 & 0.658 & 579 & 0.7806 & 0.730 & 588 & 0.7102 & 0.732 & 145 & 79 & 255 \\
\hline $\mathbf{4 - 1}$ & $\mathbf{2 6 6}$ & 0.7622 & 0.685 & 522 & 0.7620 & 0.734 & 528 & 0.7541 & 0.682 & 181 & $\mathbf{8 2}$ & $\mathbf{2 1 7}$ \\
\hline $\mathbf{4 - 2}$ & & 0.7593 & 0.689 & 514 & 0.7592 & 0.746 & 520 & 0.7483 & 0.692 & 175 & & \\
\hline $\mathbf{4 - 3}$ & & 0.7643 & 0.682 & 529 & 0.7647 & 0.729 & 536 & 0.7519 & 0.678 & 179 & & \\
\hline average & & $\mathbf{0 . 7 6 1 9}$ & $\mathbf{0 . 6 8 5}$ & $\mathbf{5 2 2}$ & $\mathbf{0 . 7 6 1 9}$ & $\mathbf{0 . 7 3 6}$ & $\mathbf{5 2 8}$ & $\mathbf{0 . 7 5 1 4}$ & $\mathbf{0 . 6 8 4}$ & $\mathbf{1 7 8}$ & & \\
\hline 5 & 233 & 0.7247 & 0.737 & 429 & 0.7241 & 0.770 & 433 & 0.7387 & 0.711 & 167 & 85 & 197 \\
\hline 6 & 203 & 0.6901 & 0.781 & 363 & 0.6888 & 0.814 & 365 & 0.7129 & 0.738 & 148 & 86 & 174 \\
\hline 7 & 182 & 0.6704 & 0.806 & 332 & 0.6710 & 0.830 & 337 & 0.6541 & 0.812 & 112 & 84 & 153 \\
\hline 8 & 164 & 0.6528 & 0.827 & 307 & 0.6530 & 0.844 & 311 & 0.7015 & 0.753 & 139 & 87 & 142 \\
\hline 9 & 152 & 0.6356 & 0.847 & 284 & 0.6348 & 0.864 & 287 & 0.6378 & 0.822 & 105 & 84 & 128 \\
\hline 10 & 139 & 0.6178 & 0.868 & 263 & 0.6218 & 0.875 & 271 & 0.6788 & 0.777 & 126 & 84 & 117 \\
\hline
\end{tabular}

\begin{tabular}{|c|c|c|c|}
\hline $\begin{array}{c}\text { Training set, } \\
\mathbf{n}=\mathbf{3 3 2}\end{array}$ & $\begin{array}{c}\text { Calibration set, } \\
\mathbf{n}=\mathbf{0}\end{array}$ & $\begin{array}{c}\text { Test set, } \\
\mathbf{n}=\mathbf{6 1}\end{array}$ & $\begin{array}{c}\mathbf{S A}_{\mathbf{k}} \\
\text { distribution }\end{array}$ \\
\hline
\end{tabular}

[Training-Test] system

\begin{tabular}{|c|c|c|c|c|c|c|c|c|c|c|c|c|}
\hline $\operatorname{limS}$ & Nact & $\mathrm{R}^{2}$ & $\mathrm{~S}$ & $\mathrm{~F}$ & $\mathrm{R}^{2}$ & $\mathrm{~s}$ & $\mathrm{~F}$ & $\mathrm{R}^{2}$ & $\mathrm{~s}$ & $\mathrm{~F}$ & $\mathrm{~W} \%$ & $\mathrm{~N}_{101}$ \\
\hline 0 & 797 & 0.8868 & 0.472 & 2593 & & & & 0.5429 & 1.002 & 71 & 47 & 376 \\
\hline 1 & 777 & 0.8851 & 0.475 & 2542 & & & & 0.5418 & 0.984 & 71 & 46 & 356 \\
\hline 2 & 542 & 0.8602 & 0.524 & 2032 & & & & 0.6042 & 0.910 & 91 & 61 & 330 \\
\hline 3 & 432 & 0.8313 & 0.576 & 1626 & & & & 0.5575 & 0.917 & 74 & 72 & 309 \\
\hline 4 & 385 & 0.8109 & 0.610 & 1417 & & & & 0.5628 & 0.910 & 76 & 75 & 289 \\
\hline 5 & 344 & 0.8007 & 0.626 & 1327 & & & & 0.5913 & 0.871 & 86 & 78 & 267 \\
\hline $\mathbf{6 - 1}$ & $\mathbf{3 1 2}$ & 0.7902 & 0.642 & 1243 & & & & 0.6744 & 0.769 & 122 & $\mathbf{8 2}$ & $\mathbf{2 5 5}$ \\
\hline $\mathbf{6 - 2}$ & & 0.7875 & 0.646 & 1223 & & & & 0.7138 & 0.721 & 147 & & \\
\hline $\mathbf{6 - 3}$ & & 0.7843 & 0.651 & 1200 & & & & 0.6947 & 0.744 & 134 & & \\
\hline average & & $\mathbf{0 . 7 8 7 3}$ & $\mathbf{0 . 6 4 7}$ & $\mathbf{1 2 2 2}$ & & & & $\mathbf{0 . 6 9 4 3}$ & $\mathbf{0 . 7 4 5}$ & $\mathbf{1 3 5}$ & & \\
\hline 7 & 288 & 0.7788 & 0.659 & 1162 & & & & 0.6579 & 0.789 & 114 & 83 & 238 \\
\hline 8 & 268 & 0.7659 & 0.678 & 1080 & & & & 0.6677 & 0.777 & 121 & 85 & 227 \\
\hline 9 & 246 & 0.7363 & 0.720 & 922 & & & & 0.6853 & 0.757 & 129 & 84 & 207 \\
\hline 10 & 234 & 0.7224 & 0.739 & 859 & & & & 0.6909 & 0.750 & 133 & 84 & 196 \\
\hline
\end{tabular}

SPLIT2

\begin{tabular}{|c|c|c|c|}
\hline $\begin{array}{c}\text { Subtraining set, } \\
\mathbf{n}=\mathbf{1 6 5}\end{array}$ & $\begin{array}{c}\text { Calibration set, } \\
\mathrm{n}=167\end{array}$ & $\begin{array}{c}\text { Test set, } \\
\mathrm{n}=\mathbf{6 1}\end{array}$ & $\begin{array}{c}\mathbf{S A}_{\mathbf{k}} \\
\text { distribution }\end{array}$ \\
\hline
\end{tabular}

[Subtraining-Calibration-Test] system Split2

\begin{tabular}{|c|c|c|c|c|c|c|c|c|c|c|c|c|}
\hline $\operatorname{limS}$ & Nact & $\mathrm{R}^{2}$ & $\mathrm{~S}$ & $\mathrm{~F}$ & $\mathrm{R}^{2}$ & $\mathrm{~S}$ & $\mathrm{~F}$ & $\mathrm{R}^{2}$ & $\mathrm{~s}$ & $\mathrm{~F}$ & $\mathrm{~W} \%$ & $\mathrm{~N}_{111}$ \\
\hline 0 & 797 & 0.8743 & 0.507 & 1134 & 0.8737 & 0.540 & 1142 & 0.4630 & 1.055 & 51 & 42 & 337 \\
\hline 1 & 632 & 0.8740 & 0.507 & 1131 & 0.8736 & 0.551 & 1140 & 0.5003 & 0.995 & 59 & 51 & 320 \\
\hline 2 & 425 & 0.8377 & 0.576 & 841 & 0.8367 & 0.580 & 846 & 0.5919 & 0.820 & 86 & 67 & 286 \\
\hline 3 & 335 & 0.8048 & 0.632 & 673 & 0.8041 & 0.633 & 678 & 0.5862 & 0.861 & 84 & 78 & 261 \\
\hline 4 & 284 & 0.7843 & 0.664 & 593 & 0.7842 & 0.663 & 600 & 0.7042 & 0.711 & 141 & 84 & 239 \\
\hline 5 & 247 & 0.7458 & 0.721 & 478 & 0.7448 & 0.728 & 482 & 0.7627 & 0.671 & 190 & 87 & 214 \\
\hline
\end{tabular}


Table 4. Cont.

\begin{tabular}{|c|c|c|c|c|c|c|c|c|c|c|c|c|}
\hline $\mathbf{6 - 1}$ & $\mathbf{2 2 4}$ & 0.7315 & 0.741 & 444 & 0.7314 & 0.748 & 449 & 0.7937 & 0.604 & 227 & $\mathbf{8 4}$ & $\mathbf{1 8 9}$ \\
\hline $\mathbf{6 - 2}$ & & 0.7234 & 0.752 & 426 & 0.7234 & 0.760 & 431 & 0.7922 & 0.605 & 225 & & \\
\hline $\mathbf{6 - 3}$ & & 0.7384 & 0.731 & 460 & 0.7384 & 0.740 & 466 & 0.8136 & 0.593 & 258 & & \\
\hline average & & $\mathbf{0 . 7 3 1 1}$ & $\mathbf{0 . 7 4 1}$ & $\mathbf{4 4 4}$ & $\mathbf{0 . 7 3 1 0}$ & $\mathbf{0 . 7 4 9}$ & $\mathbf{4 4 9}$ & $\mathbf{0 . 7 9 9 8}$ & $\mathbf{0 . 6 0 0}$ & $\mathbf{2 3 6}$ & & \\
\hline 7 & 195 & 0.6978 & 0.786 & 376 & 0.7007 & 0.781 & 386 & 0.7318 & 0.657 & 161 & 84 & 164 \\
\hline 8 & 178 & 0.6878 & 0.799 & 359 & 0.6880 & 0.801 & 364 & 0.7223 & 0.682 & 153 & 82 & 146 \\
\hline 9 & 158 & 0.6659 & 0.826 & 325 & 0.6692 & 0.831 & 334 & 0.7104 & 0.709 & 145 & 84 & 133 \\
\hline 10 & 149 & 0.6472 & 0.849 & 299 & 0.6550 & 0.847 & 313 & 0.6970 & 0.723 & 136 & 84 & 125 \\
\hline
\end{tabular}

\begin{tabular}{|c|c|c|c|}
\hline $\begin{array}{c}\text { Training set, } \\
\mathbf{n}=\mathbf{3 3 2}\end{array}$ & $\begin{array}{c}\text { Calibration set, } \\
\mathbf{N}=\mathbf{0}\end{array}$ & $\begin{array}{c}\text { Test set, } \\
\mathbf{n}=\mathbf{6 1}\end{array}$ & $\begin{array}{c}\mathbf{S A}_{\mathbf{k}} \\
\text { distribution }\end{array}$ \\
\hline
\end{tabular}

[Training-Test] system Split2

\begin{tabular}{|c|c|c|c|c|c|c|c|c|c|c|c|c|}
\hline $\operatorname{limS}$ & Nact & $\mathrm{R}^{2}$ & $\mathrm{~S}$ & $\mathrm{~F}$ & $\mathrm{R}^{2}$ & $\mathrm{~s}$ & $\mathrm{~F}$ & $\mathrm{R}^{2}$ & $\mathrm{~s}$ & $\mathrm{~F}$ & $\mathrm{~W} \%$ & $\mathrm{~N}_{101}$ \\
\hline 0 & 797 & 0.8922 & 0.468 & 2734 & & & & 0.4665 & 1.013 & 52 & 47 & 372 \\
\hline 1 & 785 & 0.8950 & 0.462 & 2815 & & & & 0.4711 & 1.029 & 53 & 46 & 360 \\
\hline 2 & 546 & 0.8740 & 0.506 & 2290 & & & & 0.5329 & 0.887 & 67 & 61 & 335 \\
\hline 3 & 442 & 0.8456 & 0.561 & 1807 & & & & 0.5767 & 0.845 & 81 & 71 & 315 \\
\hline 4 & 388 & 0.8194 & 0.606 & 1497 & & & & 0.6130 & 0.805 & 94 & 76 & 296 \\
\hline 5 & 350 & 0.8122 & 0.618 & 1428 & & & & 0.5802 & 0.873 & 82 & 79 & 278 \\
\hline 6 & 321 & 0.8103 & 0.621 & 1412 & & & & 0.6074 & 0.840 & 92 & 83 & 267 \\
\hline 7 & 287 & 0.7848 & 0.662 & 1204 & & & & 0.6689 & 0.753 & 120 & 86 & 247 \\
\hline 8 & 263 & 0.7594 & 0.700 & 1042 & & & & 0.7345 & 0.655 & 164 & 87 & 229 \\
\hline $\mathbf{9 - 1}$ & $\mathbf{2 4 3}$ & 0.7397 & 0.728 & 938 & & & & 0.7472 & 0.653 & 174 & $\mathbf{8 9}$ & $\mathbf{2 1 6}$ \\
\hline $\mathbf{9 - 2}$ & & 0.7370 & 0.732 & 925 & & & & 0.7862 & 0.602 & 217 & & \\
\hline $\mathbf{9 - 3}$ & & 0.7456 & 0.720 & 967 & & & & 0.7604 & 0.642 & 187 & & \\
\hline average & & $\mathbf{0 . 7 4 0 8}$ & $\mathbf{0 . 7 2 6}$ & $\mathbf{9 4 3}$ & & & & $\mathbf{0 . 7 6 4 6}$ & $\mathbf{0 . 6 3 2}$ & $\mathbf{1 9 3}$ & & \\
\hline 10 & 228 & 0.7294 & 0.742 & 890 & & & & 0.7502 & 0.655 & 178 & 86 & 196 \\
\hline
\end{tabular}

\section{SPLIT3}

\begin{tabular}{|c|c|c|c|}
\hline $\begin{array}{c}\text { Subtraining set, } \\
\mathbf{n}=\mathbf{1 6 5}\end{array}$ & $\begin{array}{c}\text { Calibration set, } \\
\mathbf{n}=167\end{array}$ & $\begin{array}{c}\text { Test set, } \\
\mathbf{n}=\mathbf{6 1}\end{array}$ & $\begin{array}{c}\mathbf{S A}_{\mathbf{k}} \\
\text { distribution }\end{array}$ \\
\hline
\end{tabular}

[Subtraining-Calibration-Test] system Split3

\begin{tabular}{|c|c|c|c|c|c|c|c|c|c|c|c|c|}
\hline $\operatorname{limS}$ & Nact & $\mathrm{R}^{2}$ & $\mathrm{~S}$ & $\mathrm{~F}$ & $\mathrm{R}^{2}$ & $\mathrm{~S}$ & $\mathrm{~F}$ & $\mathrm{R}^{2}$ & $\mathrm{~S}$ & $\mathrm{~F}$ & $\mathrm{~W} \%$ & $\mathrm{~N}_{111}$ \\
\hline 0 & 797 & 0.8690 & 0.518 & 1084 & 0.8909 & 0.516 & 1353 & 0.5794 & 0.929 & 82 & 42 & 332 \\
\hline 1 & 614 & 0.8742 & 0.508 & 1134 & 0.8946 & 0.513 & 1402 & 0.5995 & 0.896 & 89 & 50 & 309 \\
\hline 2 & 402 & 0.8266 & 0.597 & 778 & 0.8331 & 0.614 & 826 & 0.6748 & 0.800 & 122 & 69 & 278 \\
\hline $\mathbf{3 - 1}$ & $\mathbf{3 2 4}$ & 0.7963 & 0.647 & 637 & 0.7982 & 0.633 & 652 & 0.7176 & 0.729 & 150 & $\mathbf{7 8}$ & $\mathbf{2 5 4}$ \\
\hline $\mathbf{3 - 2}$ & & 0.7919 & 0.654 & 620 & 0.7937 & 0.639 & 635 & 0.6969 & 0.758 & 136 & & \\
\hline $\mathbf{3 - 3}$ & & 0.7930 & 0.652 & 624 & 0.7944 & 0.641 & 637 & 0.7431 & 0.698 & 171 & & \\
\hline average & & $\mathbf{0 . 7 9 3 7}$ & $\mathbf{0 . 6 5 1}$ & $\mathbf{6 2 7}$ & $\mathbf{0 . 7 9 5 4}$ & $\mathbf{0 . 6 3 8}$ & $\mathbf{6 4 2}$ & $\mathbf{0 . 7 1 9 2}$ & $\mathbf{0 . 7 2 8}$ & $\mathbf{1 5 2}$ & & \\
\hline 4 & 264 & 0.7439 & 0.725 & 474 & 0.7462 & 0.703 & 485 & 0.6992 & 0.765 & 138 & 85 & 224 \\
\hline 5 & 227 & 0.7127 & 0.768 & 404 & 0.7136 & 0.738 & 411 & 0.6900 & 0.774 & 133 & 86 & 195 \\
\hline 6 & 198 & 0.6945 & 0.792 & 371 & 0.7013 & 0.756 & 388 & 0.6899 & 0.770 & 133 & 86 & 171 \\
\hline 7 & 181 & 0.6790 & 0.812 & 345 & 0.6843 & 0.780 & 358 & 0.6995 & 0.758 & 137 & 85 & 154 \\
\hline 8 & 159 & 0.6432 & 0.856 & 294 & 0.6493 & 0.815 & 306 & 0.7061 & 0.749 & 142 & 84 & 134 \\
\hline 9 & 147 & 0.6219 & 0.881 & 268 & 0.6533 & 0.820 & 311 & 0.6934 & 0.775 & 134 & 84 & 123 \\
\hline 10 & 140 & 0.5952 & 0.911 & 240 & 0.6269 & 0.849 & 277 & 0.6300 & 0.842 & 101 & 83 & 116 \\
\hline
\end{tabular}


Table 4. Cont.

\begin{tabular}{|c|c|c|c|}
\hline $\begin{array}{c}\text { Training set, } \\
\mathbf{n}=\mathbf{3 3 2}\end{array}$ & $\begin{array}{c}\text { Calibration set, } \\
\mathbf{n}=\mathbf{0}\end{array}$ & $\begin{array}{c}\text { Test set, } \\
\mathbf{n}=\mathbf{6 1}\end{array}$ & $\begin{array}{c}\mathbf{S A}_{\mathbf{k}} \\
\text { distribution }\end{array}$ \\
\hline
\end{tabular}

[Training-Test] system Split3

\begin{tabular}{|c|c|c|c|c|c|c|c|c|c|c|c|c|}
\hline $\operatorname{limS}$ & Nact & $\mathrm{R}^{2}$ & $\mathrm{~s}$ & $\mathrm{~F}$ & $\mathrm{R}^{2}$ & $\mathrm{~s}$ & $\mathrm{~F}$ & $\mathrm{R}^{2}$ & $\mathrm{~s}$ & $\mathrm{~F}$ & $\mathrm{~W} \%$ & $\mathrm{~N}_{101}$ \\
\hline 0 & 797 & 0.8930 & 0.457 & 2756 & & & & 0.5532 & 1.009 & 73 & 47 & 377 \\
\hline 1 & 776 & 0.8932 & 0.457 & 2763 & & & & 0.5529 & 0.996 & 73 & 46 & 356 \\
\hline 2 & 540 & 0.8699 & 0.504 & 2209 & & & & 0.5998 & 0.922 & 89 & 61 & 327 \\
\hline 3 & 434 & 0.8349 & 0.568 & 1674 & & & & 0.5908 & 0.896 & 88 & 72 & 311 \\
\hline 4 & 388 & 0.8220 & 0.590 & 1528 & & & & 0.6068 & 0.865 & 92 & 75 & 291 \\
\hline 5 & 348 & 0.8030 & 0.620 & 1346 & & & & 0.6650 & 0.796 & 117 & 78 & 272 \\
\hline $\mathbf{6 - 1}$ & $\mathbf{3 2 0}$ & 0.7773 & 0.660 & 1152 & & & & 0.7017 & 0.751 & 139 & $\mathbf{8 2}$ & $\mathbf{2 6 1}$ \\
\hline $\mathbf{6 - 2}$ & & 0.7942 & 0.634 & 1273 & & & & 0.6967 & 0.761 & 136 & & \\
\hline $\mathbf{6 - 3}$ & & 0.7834 & 0.651 & 1193 & & & & 0.7171 & 0.735 & 150 & & \\
\hline average & & $\mathbf{0 . 7 8 5 0}$ & $\mathbf{0 . 6 4 8}$ & $\mathbf{1 2 0 6}$ & & & & $\mathbf{0 . 7 0 5 1}$ & $\mathbf{0 . 7 4 9}$ & $\mathbf{1 4 1}$ & & \\
\hline 7 & 288 & 0.7598 & 0.685 & 1045 & & & & 0.6807 & 0.778 & 126 & 84 & 241 \\
\hline 8 & 271 & 0.7637 & 0.679 & 1067 & & & & 0.6520 & 0.817 & 112 & 85 & 229 \\
\hline 9 & 244 & 0.7318 & 0.724 & 901 & & & & 0.6833 & 0.778 & 127 & 86 & 210 \\
\hline 10 & 232 & 0.7288 & 0.728 & 887 & & & & 0.6826 & 0.781 & 127 & 84 & 196 \\
\hline
\end{tabular}

A useful characteristic of these models is $\mathrm{W} \%=\mathrm{N}_{111} / \mathrm{Nact}$, where $\mathrm{N}_{111}$ is the number of non blocked attributes which take place in subtraining, calibration, and test set; $\mathrm{N}_{\text {act }}$ is the total number of attributes which are not blocked for a given limS. There is a correlation between $\mathrm{W} \%$ and the determination coefficient for the test set (Figure 4, Table 4). One can see from the results that good prediction ocurrs if the W\% is higher than 80 (excepting [Subtraining-Calibration-Test ] for the Split3: in this case $\mathrm{W} \%=78)$.

The model obtained in the first probe of the Monte Carlo optimization for the split 1 with limS $=4$ is calculated as follows:

$$
-\log \left(\mathrm{TD}_{50}\right)=-0.5981(0.0074)+0.1118(0.0004) * \mathrm{DCW}(4)
$$

$\mathrm{n}=165, \mathrm{r}^{2}=0.7622, \mathrm{~s}=0.685, \mathrm{~F}=522$ (subtraining set)

$\mathrm{n}=167, \mathrm{r}^{2}=0.7620, \mathrm{~s}=0.734, \mathrm{~F}=528$ (calibration set)

$\mathrm{n}=61, \mathrm{r}^{2}=0.7541, \mathrm{~s}=0.682, \mathrm{~F}=181$ (test set)

Y-scrambling $[19,20]$ for the test set $\left(\mathrm{N}_{\text {shifting }}=300[20]\right)$ gave $\mathrm{r}^{2}$ scrambling $=0.0996$

Figure 5 shows the model calculated with Equation 3, graphically. The Supplementary Materials contains numerical data on the experimental and calculated values with Equation 3 (split1 with $\operatorname{limS}=4$ ). Table 5 contains numerical data on the correlation weights of SMILES attributes obtained in three probes of the Monte Carlo optimization. 
Figure 3. Comparison of the [subtraining-calibration-test] system and the [trainingtest] system for three splits.

[ Subtraining ( $($ ) - Calibration ( 0 )-Test ( 4 )] system
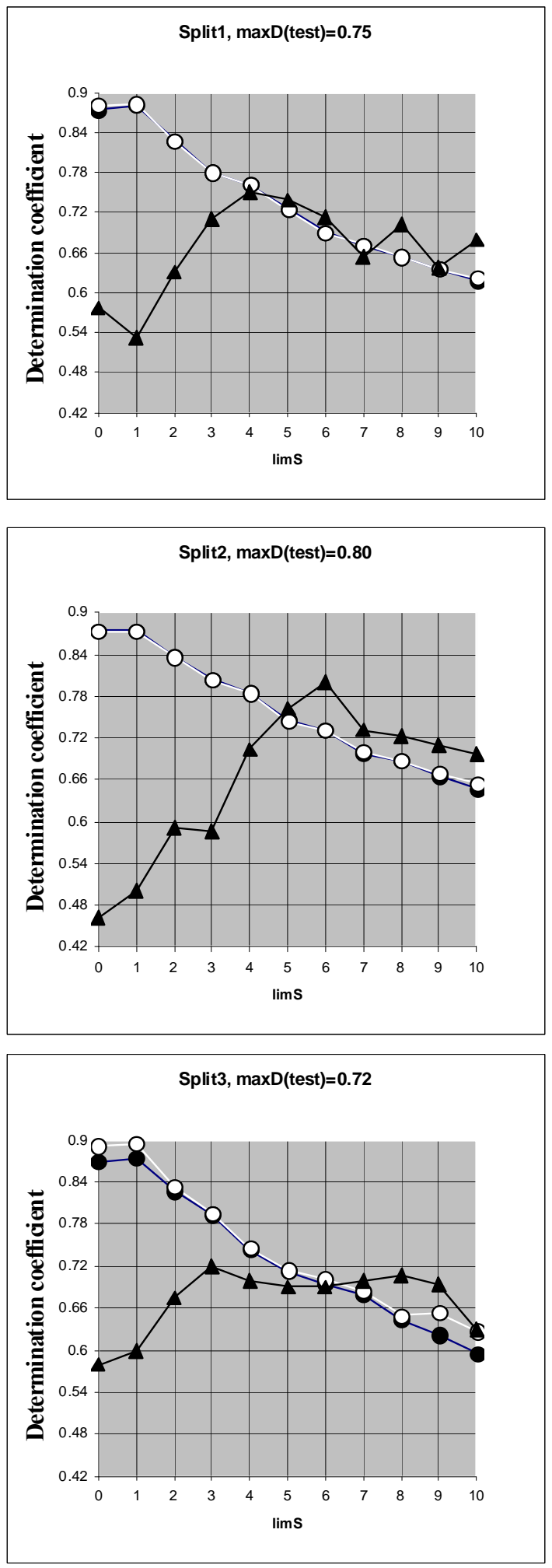

[ Training (*)-Test ( $*$ )] system
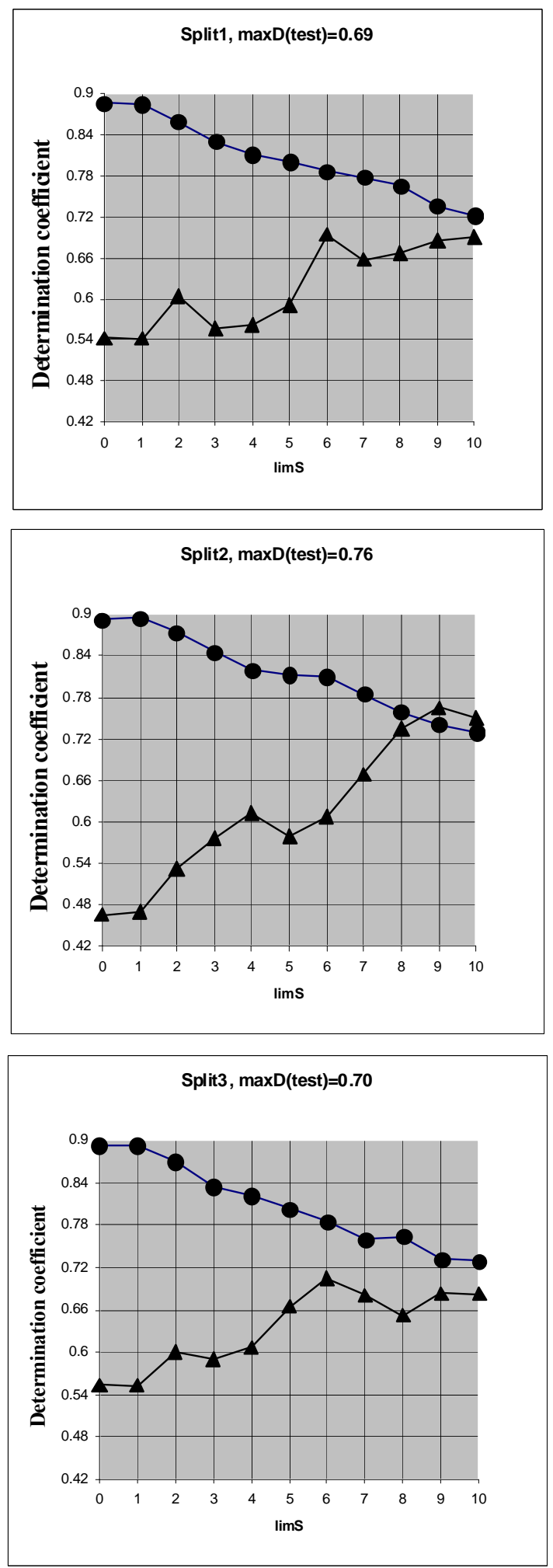
Figure 4. Correlations between the determination coefficient for test set and $\mathrm{W} \%$ for the three splits (see data from Table 4).

[Subtraining-Calibration-Test] system

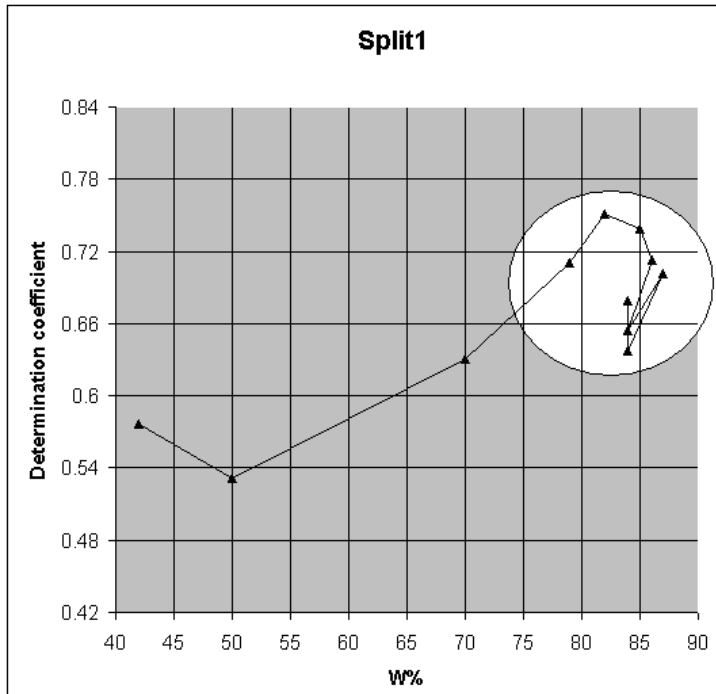

Split2

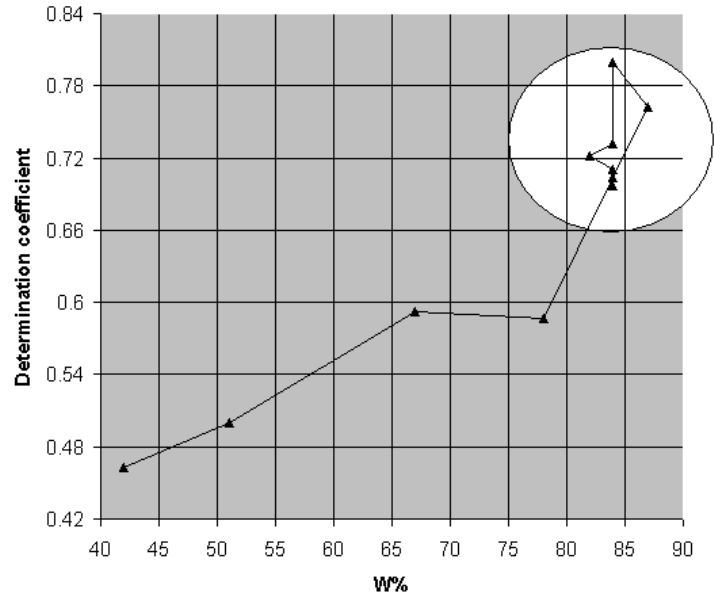

Split3

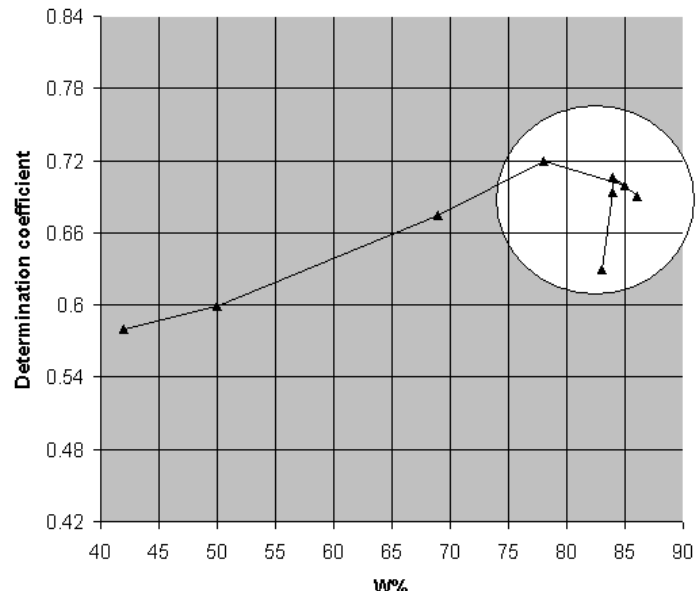

[Training-Test] system
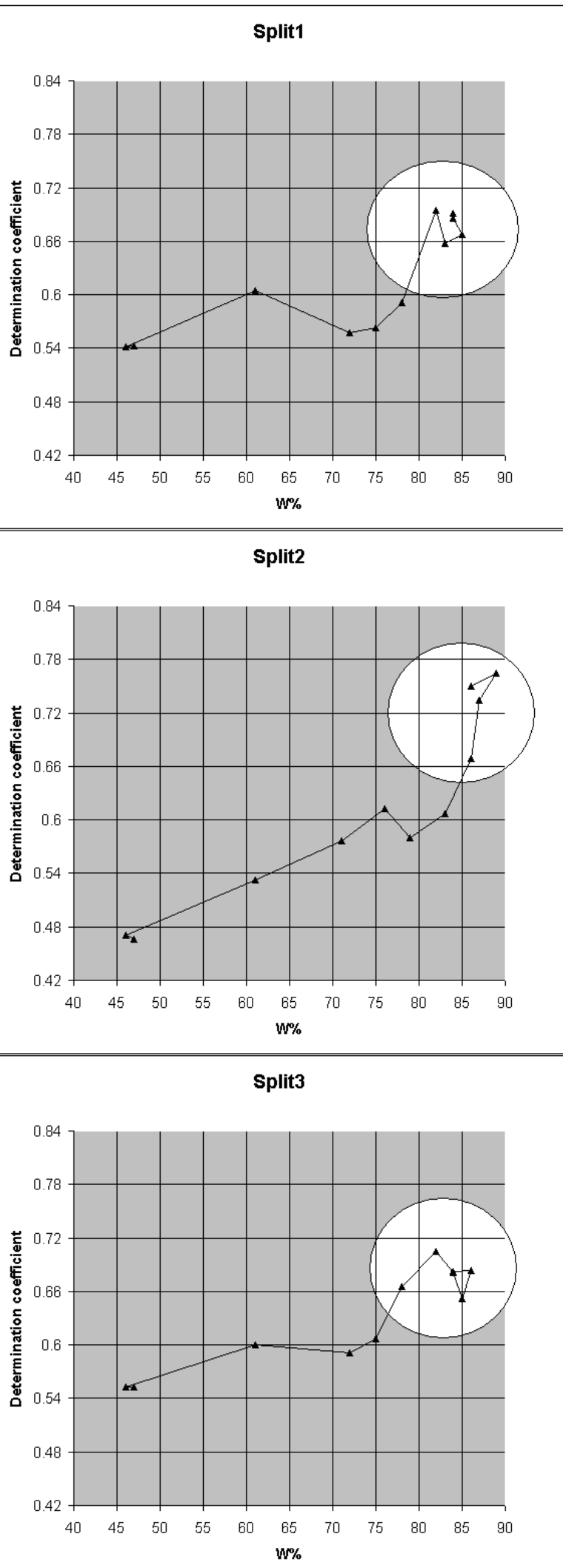
Table 5. Correlation weights for calculation with Equation 1 DCW(4). N(Subtr), N(calib), and N(Test) are numbers of a given SMILES attribute in the subtraining set, calibration set, and test set, respectively. The rare attributes are omitted.

\begin{tabular}{|c|c|c|c|c|c|c|}
\hline $\begin{array}{l}\text { SMILES-Attributes } \\
\text { (SA) }\end{array}$ & $\begin{array}{l}\text { CW(SA) } \\
\text { probe } 1\end{array}$ & $\begin{array}{l}\text { CW(SA) } \\
\text { probe } 2\end{array}$ & $\begin{array}{l}\text { CW(SA) } \\
\text { probe } 3\end{array}$ & $\begin{array}{c}\text { N(Subtr } \\
\text { ) }\end{array}$ & $\begin{array}{c}\text { N(Calib } \\
\text { ) }\end{array}$ & $\begin{array}{c}\text { N(Test } \\
\text { ) }\end{array}$ \\
\hline \multicolumn{7}{|c|}{$\mathrm{dC}$} \\
\hline !-01_........ & 2.7522274 & 2.8704615 & 3.5346711 & 5 & 4 & 0 \\
\hline$!-02 \ldots \ldots \ldots$ & 1.2190257 & 2.1277910 & 1.8790680 & 10 & 9 & 3 \\
\hline$!-03 \ldots \ldots \ldots$ & 6.6784389 & 8.0311759 & 7.1271958 & 15 & 10 & 3 \\
\hline !-04........ & 1.4326102 & 1.6702225 & 1.9340790 & 17 & 22 & 8 \\
\hline$!-05 \ldots \ldots \ldots$ & 3.9671055 & 4.0344924 & 4.1729635 & 9 & 11 & 6 \\
\hline !-06......... & 5.8564637 & 5.8794012 & 6.4409754 & 8 & 11 & 7 \\
\hline !-07........ & 5.4970475 & 5.1611240 & 5.2308474 & 5 & 3 & 0 \\
\hline$!-08 \ldots \ldots .$. & 9.1295923 & 9.5122328 & 9.0035813 & 4 & 3 & 1 \\
\hline$!-21 \ldots \ldots$. & -1.6383248 & 1.8781962 & 0.0037831 & 4 & 0 & 0 \\
\hline !000......... & 3.6271821 & 4.6894405 & 3.7495506 & 6 & 7 & 1 \\
\hline !002.......... & 1.5603260 & 1.7450611 & 1.4951171 & 4 & 4 & 1 \\
\hline !003......... & -1.2514096 & -1.3248590 & -1.1256941 & 5 & 8 & 2 \\
\hline !004......... & 0.7359726 & 1.0643522 & 1.2450258 & 11 & 8 & 1 \\
\hline !005 ......... & 0.9702817 & 0.6240636 & 1.2144260 & 13 & 9 & 4 \\
\hline !006......... & 4.1543029 & 4.9338830 & 4.9975361 & 7 & 5 & 2 \\
\hline !007......... & -3.7770327 & -3.5039029 & -3.0945823 & 4 & 3 & 3 \\
\hline !010......... & 0.5049355 & -0.2636435 & 0.3157527 & 6 & 8 & 3 \\
\hline !012......... & 3.2511213 & 3.2471578 & 4.5049864 & 6 & 3 & 1 \\
\hline \multicolumn{7}{|l|}{${ }^{1} \mathbf{S A}_{\mathbf{k}}$} \\
\hline \#............. & 3.3706294 & 3.3739877 & 2.0643948 & 5 & 3 & 0 \\
\hline$(\ldots \ldots \ldots \ldots$ & -1.6866726 & -1.3666396 & -1.5485382 & 708 & 780 & 260 \\
\hline |............. & -0.4913426 & 0.1880630 & -1.0975733 & 17 & 24 & 4 \\
\hline $1 \ldots \ldots \ldots \ldots$ & -1.4970879 & -0.8440743 & -0.0771659 & 222 & 222 & 88 \\
\hline $2 \ldots \ldots \ldots . . .$. & -0.1050677 & -1.1891334 & -1.1138329 & 130 & 132 & 48 \\
\hline 3............... & -1.3433340 & 0.0456678 & -0.1828115 & 60 & 60 & 20 \\
\hline $4 \ldots \ldots \ldots \ldots$. & 3.4954870 & 3.1562107 & 3.5453447 & 20 & 18 & 8 \\
\hline $5 \ldots \ldots \ldots \ldots .$. & 2.8128037 & 3.3899959 & 1.6902086 & 10 & 10 & 4 \\
\hline$=\ldots \ldots \ldots \ldots$ & -1.8660845 & -2.1441609 & -1.8865449 & 77 & 79 & 23 \\
\hline С................ & -0.0156855 & 0.0453525 & 0.2198595 & 765 & 736 & 290 \\
\hline $\operatorname{Br} \ldots . . . . . . .$. & 0.5327181 & 0.2779344 & 0.8454938 & 23 & 8 & 1 \\
\hline $\mathrm{Cl}$................ & 2.9838590 & 2.1906970 & 3.1890603 & 61 & 85 & 13 \\
\hline F............... & -0.4680666 & -1.0425952 & 0.2836492 & 15 & 19 & 8 \\
\hline $\mathrm{O}=\mathrm{C} \ldots \ldots \ldots .$. & -2.8475657 & -2.4376628 & -2.9332073 & 33 & 21 & 13 \\
\hline $\mathrm{O}=\ldots \ldots \ldots \ldots$ & 0.7369372 & 0.0037805 & 1.4086398 & 140 & 132 & 47 \\
\hline N................. & 1.1227501 & 1.1982965 & 1.4193640 & 196 & 201 & 76 \\
\hline $\mathrm{O}, \ldots \ldots \ldots \ldots$ & -1.2649109 & -0.4408418 & -0.1501499 & 138 & 143 & 45 \\
\hline S............. & 2.3712714 & 2.6251760 & 2.5313565 & 13 & 12 & 7 \\
\hline$[\mathrm{N}+] \ldots \ldots$. & 1.9345689 & 1.6543771 & 3.0457447 & 26 & 31 & 12 \\
\hline$[\mathrm{O}-] . \ldots \ldots .$. & 5.9250900 & 5.6230564 & 6.9653600 & 26 & 32 & 12 \\
\hline$[\ldots \ldots \ldots \ldots$. & -2.1531745 & -2.8080919 & -1.9966710 & 4 & 6 & 0 \\
\hline \............. & 3.3565892 & 3.4338813 & 2.9027414 & 14 & 29 & 7 \\
\hline c............. & -0.0357264 & 0.0373181 & 0.0419142 & 653 & 679 & 247 \\
\hline $\mathrm{n} . \ldots \ldots \ldots . . .$. & -0.6564241 & -0.1251570 & -1.4184164 & 37 & 44 & 23 \\
\hline $0 \ldots \ldots \ldots \ldots . .$. & -1.0665085 & -1.3777640 & -0.2485470 & 16 & 12 & 7 \\
\hline & -0.0527175 & -0.9991370 & -1.0040993 & 7 & 6 & 7 \\
\hline \multicolumn{7}{|l|}{${ }^{2} \mathbf{S A}_{\mathrm{k}}$} \\
\hline$(\ldots(\ldots \ldots)$. & -0.0735964 & -0.1751550 & -0.4970432 & 18 & 28 & 4 \\
\hline |...(........ & -0.9972903 & -1.5270762 & -0.7479799 & 7 & 10 & 2 \\
\hline $1 \ldots(\ldots \ldots$. & 2.3733452 & 2.4975765 & 2.4084744 & 37 & 45 & 15 \\
\hline $2 \ldots(\ldots \ldots \ldots$ & 0.0608136 & 0.1227718 & -0.1848792 & 14 & 15 & 6 \\
\hline $2 \ldots 1 \ldots$ & 5.7529509 & 6.6287931 & 7.4713129 & 5 & 6 & 2 \\
\hline
\end{tabular}


Table 5. Cont.

\begin{tabular}{|c|c|c|c|c|c|c|}
\hline $3 \ldots(\ldots \ldots .$. & -1.6283079 & -0.2528134 & -2.0499858 & 6 & 3 & 0 \\
\hline $3 \ldots 2 \ldots \ldots$ & -1.5915226 & -1.5268568 & -1.9365673 & 4 & 6 & 3 \\
\hline$=\ldots(\ldots \ldots \ldots$ & 1.8468333 & 2.3079839 & 2.4377851 & 14 & 17 & 3 \\
\hline$=\ldots 1 \ldots \ldots$ & 2.5039102 & -0.5608527 & -0.0325572 & 7 & 5 & 1 \\
\hline$=\ldots 2 \ldots \ldots$ & -3.2847055 & -2.5042239 & -2.0658154 & 7 & 5 & 2 \\
\hline$=\ldots 3 \ldots \ldots$ & 3.4389111 & 0.8278003 & 3.5625409 & 6 & 5 & 4 \\
\hline С...\#........ & -0.1836222 & -0.9385750 & -0.4107562 & 6 & 3 & 0 \\
\hline С...(........ & -0.7573851 & 0.0280162 & 0.7466835 & 443 & 456 & 163 \\
\hline С.../....... & 1.1245359 & 0.0042443 & 0.8873189 & 13 & 13 & 2 \\
\hline С...1....... & -0.4566262 & 0.0357389 & -1.3911620 & 74 & 73 & 30 \\
\hline С...2....... & 0.3115003 & 1.0911890 & 0.8401947 & 46 & 47 & 12 \\
\hline С...3........ & 3.6534836 & 3.4678053 & 2.9866499 & 40 & 22 & 8 \\
\hline С...4....... & -0.7152591 & -0.7967591 & -1.2502575 & 17 & 13 & 5 \\
\hline С...5 ........ & 3.6909807 & 4.4229382 & 4.3015822 & 10 & 7 & 6 \\
\hline $\mathrm{C} \ldots=\ldots \ldots$ & -0.5319093 & -0.5807285 & -0.4569253 & 98 & 101 & 29 \\
\hline С...С........ & -0.4098212 & -0.6663667 & -0.4722713 & 244 & 211 & 113 \\
\hline Br..(......... & -1.2467411 & -0.7804139 & -0.9676602 & 24 & 7 & 0 \\
\hline Br..C........ & 5.8039394 & 6.6601591 & 5.9721683 & 9 & 5 & 1 \\
\hline Cl..(......... & -0.2165917 & -0.6443513 & -0.7389015 & 68 & 104 & 11 \\
\hline Cl..C......... & 6.8768666 & 7.6839570 & 7.4343341 & 17 & 18 & 5 \\
\hline F...(....... & 0.2020867 & -0.0538118 & -0.1868874 & 24 & 22 & 12 \\
\hline $\mathrm{O}=\mathrm{C} .(\ldots \ldots .$. & 0.7311485 & -1.6257029 & 0.2188934 & 18 & 8 & 5 \\
\hline $\mathrm{O}=\mathrm{C} .1 \ldots \ldots \ldots$ & 4.3778160 & 4.7809340 & 4.0011131 & 9 & 6 & 1 \\
\hline $\mathrm{O}=. .(\ldots \ldots .$. & -0.5612999 & -1.5272716 & -1.1454337 & 177 & 158 & 60 \\
\hline $\mathrm{O}=. .1 \ldots \ldots$. & -2.5019413 & -3.3715192 & -4.0028237 & 4 & 2 & 0 \\
\hline N...\#........ & -3.8725309 & -4.4992215 & -3.7843832 & 4 & 2 & 0 \\
\hline N...(....... & 0.0666245 & 0.7453778 & -0.1289674 & 140 & 165 & 56 \\
\hline N.../........ & 0.8133323 & 0.0606093 & -0.1893841 & 9 & 12 & 2 \\
\hline N...1....... & 1.8744868 & 1.0335496 & 1.5038557 & 23 & 17 & 10 \\
\hline N...2....... & 1.4979132 & 1.4959901 & 1.4961647 & 6 & 9 & 3 \\
\hline $\mathrm{N}_{\ldots} \ldots=\ldots \ldots$ & -1.3157419 & 0.1537739 & -0.3882898 & 12 & 16 & 5 \\
\hline N...C........ & 1.4051238 & 0.9827410 & 1.0619180 & 63 & 70 & 24 \\
\hline $\mathrm{N} \ldots \mathrm{O}=\ldots \ldots$ & 6.1270291 & 7.3000058 & 4.8170313 & 39 & 34 & 13 \\
\hline N...N........ & 3.1922498 & 3.5013150 & 4.1321688 & 14 & 8 & 8 \\
\hline O.............. & -0.1195150 & -0.1976562 & -0.7838811 & 106 & 111 & 31 \\
\hline О...1....... & -0.7620380 & -1.4388311 & -1.9361803 & 19 & 13 & 5 \\
\hline O...2....... & -2.5618134 & -3.2668394 & -2.8747322 & 9 & 5 & 3 \\
\hline О...С........ & 1.0444154 & 1.0339726 & 0.9105754 & 90 & 96 & 29 \\
\hline S...(....... & -0.7479741 & 0.4990928 & -0.0132133 & 7 & 8 & 4 \\
\hline $\mathrm{S} . . .=\ldots \ldots$ & 1.5009045 & 0.6752299 & -0.2807681 & 5 & 7 & 2 \\
\hline S...C........ & 0.2470117 & -1.2535030 & -0.5349209 & 6 & 1 & 4 \\
\hline$[\mathrm{N}+](\ldots \ldots$. & 3.2516821 & 1.6524330 & 1.3748828 & 40 & 37 & 17 \\
\hline$[\mathrm{O}-](\ldots \ldots \ldots$ & -0.4532482 & -0.8221590 & -1.3547359 & 39 & 48 & 18 \\
\hline$[\mathrm{O}-][\mathrm{N}+] \ldots$ & 0.2616708 & 0.6284804 & -1.2536848 & 5 & 6 & 2 \\
\hline \...(........ & 0.2506876 & -0.8700648 & -1.1268254 & 5 & 11 & 1 \\
\hline \...C........ & 2.1710329 & 2.6262343 & 1.7619375 & 11 & 26 & 6 \\
\hline \...N........ & -3.1201815 & -3.8706242 & -3.0325970 & 4 & 12 & 3 \\
\hline c...(....... & 0.3275817 & -0.1910585 & -0.5343311 & 183 & 238 & 94 \\
\hline c...1...... & 0.5127781 & 0.1714980 & 0.8236519 & 196 & 204 & 75 \\
\hline c...2...... & 0.1139969 & 1.4331593 & 2.2509927 & 129 & 122 & 38 \\
\hline c...3....... & 1.5045372 & 1.4375414 & 0.1592669 & 41 & 50 & 15 \\
\hline c...4...... & 0.9391582 & 0.2451376 & 0.7605772 & 9 & 10 & 10 \\
\hline с ...С ........ & -1.6459258 & 0.0580657 & -0.4333240 & 15 & 19 & 1 \\
\hline с...Cl....... & -1.9973422 & -2.7517912 & -3.6905785 & 5 & 7 & 3 \\
\hline c...N........ & 1.0896408 & 0.1897391 & 0.7548234 & 26 & 19 & 12 \\
\hline с...О......... & 2.4331156 & 1.2515997 & 0.9178503 & 22 & 18 & 6 \\
\hline с...c....... & -0.2252497 & -0.6284915 & -0.8624749 & 316 & 305 & 106 \\
\hline
\end{tabular}


Table 5. Cont.

\begin{tabular}{|c|c|c|c|c|c|c|}
\hline $\mathrm{n}$ n...(........... & 0.8765637 & -0.9401023 & 0.2494319 & 11 & 8 & 6 \\
\hline n...1....... & 1.6235455 & 1.2765370 & 1.8586068 & 16 & 15 & 10 \\
\hline п...2....... & 2.0303385 & 3.9797145 & 3.5009942 & 6 & 11 & 8 \\
\hline n...3....... & 4.1295873 & 3.5576218 & 4.1220666 & 4 & 6 & 3 \\
\hline n...c........ & 2.3101715 & 1.5599987 & 1.7164852 & 25 & 40 & 17 \\
\hline o...(....... & -3.9990305 & -2.9953875 & -3.7613936 & 8 & 9 & 6 \\
\hline $0 \ldots 1 \ldots \ldots$ & 5.8875962 & 7.1857177 & 6.8437068 & 5 & 5 & 2 \\
\hline $0 . .2 \ldots \ldots$ & -0.6199000 & -0.1044473 & -0.2658548 & 7 & 6 & 5 \\
\hline о...c........ & 5.5725605 & 4.2549084 & 3.0289124 & 8 & 3 & 1 \\
\hline s...1....... & 0.9407152 & 1.9765325 & 2.0040980 & 6 & 6 & 7 \\
\hline S...c........ & -0.4960014 & 0.0005352 & 0.0003709 & 4 & 2 & 6 \\
\hline \multicolumn{7}{|l|}{${ }^{3} \mathbf{S A}_{\mathrm{k}}$} \\
\hline$(\ldots \mathrm{C} \ldots(\ldots)$ & 3.9980286 & 3.5630634 & 2.4208800 & 95 & 102 & 42 \\
\hline (...Br...(... & 0.0004754 & 0.5262619 & -1.7321140 & 9 & 3 & 0 \\
\hline (...Cl...... & 0.6233843 & -0.3777470 & 1.3569611 & 29 & 44 & 5 \\
\hline$(\ldots F \ldots(\ldots)$ & 1.9216525 & 2.4994253 & 1.1337512 & 11 & 9 & 5 \\
\hline$(\ldots \mathrm{O}=. .(\ldots$ & 1.2586684 & 0.9193678 & 0.2775945 & 71 & 68 & 28 \\
\hline$(\ldots N \ldots)(\ldots$ & 2.1549141 & 1.5500875 & 1.3741232 & 29 & 40 & 11 \\
\hline$(\ldots O \ldots(\ldots)$ & 1.3921586 & 0.5590769 & 0.3741692 & 33 & 34 & 8 \\
\hline$(\ldots[\mathrm{N}+](\ldots$ & 2.2543551 & 4.4491588 & 4.0323477 & 15 & 8 & 5 \\
\hline$(\ldots[\mathrm{O}-](\ldots$ & -1.5354823 & -1.4737180 & -3.3779724 & 19 & 23 & 9 \\
\hline$(\ldots . . . . . .$. & -1.0930229 & -0.9342219 & 0.4332127 & 12 & 18 & 0 \\
\hline /...С....... & 4.4970818 & 4.0048287 & 3.0000737 & 5 & 4 & 0 \\
\hline $1 \ldots$....... & 4.2142525 & 2.8161885 & 3.1850279 & 16 & 16 & 5 \\
\hline 1...О...... & 2.2528410 & 0.3797661 & 1.4952742 & 4 & 2 & 0 \\
\hline $1 \ldots c \ldots(\ldots$ & 0.9335129 & 0.5288083 & 0.8716499 & 18 & 35 & 12 \\
\hline $2 \ldots C \ldots(\ldots$ & -2.2544539 & -1.0919167 & -2.6427067 & 10 & 13 & 4 \\
\hline $2 \ldots . . .(\ldots$ & 2.9972422 & 3.4417285 & 3.8400379 & 29 & 28 & 12 \\
\hline $2 \ldots c \ldots 1 \ldots$ & 1.9960734 & 0.5619955 & -0.0932258 & 7 & 8 & 2 \\
\hline $2 \ldots 0 \ldots(\ldots$ & 1.0110528 & 1.4973281 & 1.0603210 & 4 & 4 & 4 \\
\hline 3...C....... & -2.2814415 & -2.8156671 & -1.9977117 & 7 & 6 & 1 \\
\hline $3 \ldots$ С...2... & 6.4980735 & 8.0020020 & 8.2529271 & 4 & 0 & 0 \\
\hline 3...c...(... & -0.2171379 & 1.0578016 & 1.2483687 & 9 & 9 & 4 \\
\hline $3 \ldots$ c...2 $\ldots$ & 5.2502023 & 4.2226554 & 4.7487134 & 8 & 7 & 2 \\
\hline 4...С...(... & 1.7464204 & -0.6210801 & -0.2842473 & 6 & 4 & 0 \\
\hline$=\ldots$ C...3 $\ldots$ & 7.0029958 & 7.5641676 & 6.7549811 & 8 & 4 & 0 \\
\hline$=\ldots C \ldots 1 \ldots$ & 2.9999456 & 2.8474909 & 4.2545986 & 12 & 8 & 4 \\
\hline$=\ldots C \ldots(\ldots$ & 1.5713076 & 0.9521538 & 0.5309943 & 18 & 18 & 2 \\
\hline$=\ldots C \ldots / \ldots$ & 5.2495800 & 5.4994084 & 6.0049990 & 6 & 7 & 2 \\
\hline$=\ldots \mathrm{N} . . . / \ldots$ & 5.8119346 & 5.5954944 & 6.3789368 & 7 & 8 & 2 \\
\hline С...(... $\ldots$ & 0.5513380 & -0.2378199 & -1.0864076 & 69 & 64 & 24 \\
\hline С...(...1... & -1.1216831 & -3.3768328 & -2.4951909 & 9 & 10 & 3 \\
\hline С...(... $=\ldots$ & 6.2535976 & 5.1587497 & 4.4360925 & 9 & 11 & 3 \\
\hline С...(...... & -0.3146880 & -1.6918223 & -1.2834724 & 11 & 22 & 3 \\
\hline С.../....... & -3.0637430 & -1.8160468 & -2.9987839 & 5 & 4 & 1 \\
\hline С...1...С... & 5.4954661 & 3.9189550 & 4.6140280 & 8 & 10 & 5 \\
\hline С...1...... & 1.3718529 & 1.3079333 & 1.4795097 & 8 & 13 & 1 \\
\hline С...1... $=\ldots$ & 0.2476856 & 1.3169467 & 0.9333222 & 6 & 4 & 1 \\
\hline С...2...... & -0.6449419 & -0.8430901 & -0.7370108 & 5 & 9 & 0 \\
\hline С...2...С... & 5.9965379 & 5.9966533 & 6.0017257 & 8 & 6 & 1 \\
\hline C...2... $=\ldots$ & 0.0028591 & -0.8747150 & -0.3764369 & 7 & 5 & 2 \\
\hline С...3...... & 6.5045056 & 6.2477966 & 5.7492216 & 5 & 3 & 0 \\
\hline C...3... $=\ldots$ & 5.6231609 & 6.0002916 & 5.1293498 & 5 & 3 & 2 \\
\hline С...3...С... & -3.5000076 & -2.9954231 & -3.0028309 & 11 & 2 & 3 \\
\hline С...4...С $\ldots$ & -3.0021372 & -4.5016046 & -2.9968826 & 4 & 1 & 1 \\
\hline C... $=\ldots 1 \ldots$ & 0.4331526 & 2.6582434 & 1.9050556 & 7 & 5 & 1 \\
\hline
\end{tabular}


Table 5. Cont.

\begin{tabular}{|c|c|c|c|c|c|c|}
\hline $\mathrm{C} \ldots=\ldots(\ldots$ & -2.4953051 & -0.6283873 & -0.7473193 & 8 & 11 & 1 \\
\hline C $\ldots=\ldots C_{\ldots}$ & 1.6093540 & 2.7975919 & 2.1897927 & 33 & 34 & 11 \\
\hline $\mathrm{C}_{\ldots} \ldots=\ldots 3 \ldots$ & 0.2971809 & 1.5013043 & -0.9639603 & 5 & 3 & 2 \\
\hline C... $=\ldots 2 \ldots$ & 1.6275637 & 3.7172258 & 1.7773288 & 7 & 4 & 0 \\
\hline С...С $\ldots 3 \ldots$ & 5.0746157 & 4.6119002 & 4.5908880 & 16 & 8 & 5 \\
\hline С...С $\ldots=\ldots$ & -0.4018420 & -1.0583619 & -1.0977818 & 36 & 26 & 5 \\
\hline 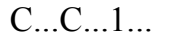 & 1.8775937 & 0.9384077 & 1.3828674 & 31 & 27 & 17 \\
\hline С...С...2... & 0.7079969 & -0.1879112 & 0.6274962 & 21 & 19 & 3 \\
\hline С...С ...... & 1.0123078 & 0.7924398 & 0.4047303 & 109 & 109 & 42 \\
\hline С...С $\ldots 4 \ldots$ & -1.4024533 & -0.7803070 & -0.3715353 & 7 & 5 & 2 \\
\hline С...С...С... & -0.0428402 & 0.1882515 & -0.1515135 & 77 & 58 & 59 \\
\hline С...Br..(... & 1.6298040 & 2.5038504 & 1.0267176 & 4 & 1 & 0 \\
\hline С...Cl..(... & -1.4962815 & -0.4993582 & -1.2516847 & 4 & 4 & 1 \\
\hline C...N...1... & -0.2691219 & -0.3795019 & -1.0000894 & 8 & 8 & 1 \\
\hline C...N...(... & 1.4422504 & 0.9731136 & 0.4339815 & 36 & 35 & 18 \\
\hline С...О...2... & 5.1236472 & 4.0895698 & 3.5121038 & 5 & 3 & 1 \\
\hline С...О...(... & 3.2515162 & 2.2843408 & 2.5780816 & 28 & 32 & 12 \\
\hline С...О...С $\ldots$ & 4.3741698 & 4.3105041 & 3.0634685 & 8 & 10 & 4 \\
\hline С...О...1... & 2.8733789 & 3.1267319 & 2.9673439 & 13 & 9 & 3 \\
\hline С...І...С... & -2.8461979 & -3.8759584 & -2.2789129 & 4 & 8 & 1 \\
\hline С...c...2... & 6.0006860 & 4.5315715 & 5.2468516 & 4 & 5 & 0 \\
\hline С...c...1... & 2.4356912 & 0.4351720 & 1.2472872 & 10 & 13 & 1 \\
\hline Br...(... ... & 2.0615855 & 1.1437599 & 1.9040185 & 4 & 6 & 0 \\
\hline Br..C...(... & 1.4981969 & 0.6256406 & 0.0009660 & 7 & 3 & 0 \\
\hline Cl......... & -1.2075807 & -0.6362162 & -1.9255180 & 9 & 6 & 0 \\
\hline Cl...(... ... & -1.1526609 & -0.2476848 & -1.8147825 & 27 & 32 & 4 \\
\hline Cl... ...Cl.. & 0.5049208 & 3.2539441 & 0.6886852 & 4 & 7 & 0 \\
\hline Сl..С...С... & -0.0014586 & 0.0039516 & -1.2464369 & 9 & 10 & 2 \\
\hline Cl..c...1... & -0.2533902 & 1.6295626 & 2.2512123 & 4 & 6 & 3 \\
\hline F......... & 1.6863754 & 1.5015167 & 0.5346605 & 5 & 8 & 2 \\
\hline F...(...(... & -1.7457403 & -2.1139078 & -1.1982770 & 6 & 8 & 4 \\
\hline $\mathrm{O}=\mathrm{C} .1 \ldots \mathrm{C} \ldots$ & 5.1279970 & 2.8669862 & 3.6914477 & 4 & 3 & 1 \\
\hline $\mathrm{O}=. .(\ldots \mathrm{C} \ldots$ & 0.8107109 & 0.7780984 & -0.4033174 & 92 & 68 & 31 \\
\hline $\mathrm{O}=. .1 \ldots \mathrm{C} \ldots$ & -3.7510578 & -4.1255552 & -4.1222938 & 4 & 2 & 0 \\
\hline $\mathrm{O}=. . \mathrm{N} \ldots(\ldots$ & 9.5435183 & 10.0636543 & 10.0315899 & 24 & 28 & 6 \\
\hline N...\#...C... & -4.5000318 & -4.5014930 & -4.5004055 & 4 & 1 & 0 \\
\hline N...(..N ... & 1.1916803 & 0.9983579 & 1.6288201 & 12 & 10 & 2 \\
\hline N...(...1... & -0.1264931 & -0.7538114 & 0.0454142 & 5 & 7 & 0 \\
\hline N...(...C... & 3.5018836 & 2.2822748 & 3.0336305 & 55 & 62 & 30 \\
\hline $\mathrm{N} \ldots(\ldots \mathrm{O}=.$. & -2.3138510 & -2.3145054 & -1.5031092 & 23 & 14 & 5 \\
\hline $\mathrm{N} \ldots(\ldots \mathrm{O}=\mathrm{C}$. & -1.4990141 & -0.8704228 & -1.2494712 & 6 & 5 & 2 \\
\hline N...1...C... & 2.6914072 & 2.5647275 & 2.7184638 & 12 & 13 & 4 \\
\hline $\mathrm{N} \ldots . . .2 \ldots \mathrm{C} \ldots$ & -0.4978517 & -0.0000051 & 1.0021092 & 5 & 6 & 1 \\
\hline N...C...(... & -0.8104915 & -0.4341890 & -0.9684013 & 25 & 24 & 8 \\
\hline …С...С... & -1.2520104 & -0.7226801 & -1.0008159 & 22 & 26 & 6 \\
\hline $\mathrm{N} \ldots \mathrm{O}=. .(\ldots$ & 2.6715972 & 1.6272171 & 3.6550784 & 11 & 11 & 1 \\
\hline N...N...1... & 0.0042593 & 1.4970138 & 1.1825822 & 5 & 3 & 3 \\
\hline $\mathrm{N} \ldots \mathrm{N} \ldots \mathrm{O}=\ldots$ & 4.2459870 & 3.7466101 & 3.2221499 & 10 & 6 & 5 \\
\hline N...N...(... & 4.7536740 & 5.4055258 & 4.6294702 & 6 & 4 & 2 \\
\hline $\mathrm{N} \ldots . . . . .2 \ldots$ & -3.6269860 & -2.8792235 & -4.2532265 & 5 & 3 & 1 \\
\hline N...c...1... & -0.1899251 & 0.2338572 & 0.2627307 & 20 & 15 & 11 \\
\hline $\mathrm{O} \ldots(\ldots \mathrm{O}=.$. & -0.6219316 & 1.4395827 & 1.2532594 & 19 & 17 & 5 \\
\hline О...(... ... & 0.9395840 & -0.4347300 & 0.4467587 & 52 & 40 & 17 \\
\hline O......(... & 11.5040533 & 12.0049318 & 11.9989881 & 4 & 4 & 1 \\
\hline $\mathrm{O} \ldots(\ldots \mathrm{O}=\mathrm{C}$. & 4.9368982 & 4.9994000 & 4.7472261 & 7 & 2 & 0 \\
\hline О..С...1... & -0.4987416 & 0.4978181 & 0.9413176 & 4 & 4 & 3 \\
\hline
\end{tabular}


Table 5. Cont.

\begin{tabular}{|c|c|c|c|c|c|c|}
\hline O_..С...С... & -2.6559832 & -3.2412835 & -3.2024047 & 35 & 37 & 10 \\
\hline О...С....... & -0.1201364 & -0.3106109 & -1.2477221 & 27 & 31 & 10 \\
\hline О...c...1... & -2.7623334 & -2.0920952 & -2.1242060 & 14 & 8 & 3 \\
\hline О...c...2.. & -1.4980614 & -3.5286748 & -3.6223778 & 7 & 6 & 0 \\
\hline S...C...C... & -0.0034408 & 1.5042803 & 0.7537994 & 4 & 0 & 2 \\
\hline$[\mathrm{N}+](\ldots \mathrm{C} \ldots$ & 9.2539066 & 9.4417355 & 7.5006239 & 6 & 4 & 1 \\
\hline$[\mathrm{N}+](\ldots 2 \ldots$ & 5.4109375 & 4.6273127 & 3.2821267 & 6 & 3 & 4 \\
\hline$[\mathrm{N}+](\ldots \mathrm{O}=.$. & -0.3787790 & 0.3109436 & 0.1872916 & 4 & 7 & 2 \\
\hline$[\mathrm{O}-](\ldots[\mathrm{N}+]$ & -3.8743809 & -1.4388021 & -1.2453181 & 18 & 22 & 9 \\
\hline$[\mathrm{O}-](\ldots \mathrm{O}=.$. & -4.0585677 & -2.6242009 & -2.5577359 & 15 & 11 & 6 \\
\hline$[\mathrm{O}-][\mathrm{N}+](\ldots$ & -3.5045096 & -1.4982980 & -2.4892797 & 5 & 5 & 2 \\
\hline$\backslash \ldots C \ldots=\ldots$ & -1.3136029 & -1.8755430 & -1.2854492 & 4 & 11 & 1 \\
\hline \...С...(... & -3.5018378 & -4.4994516 & -3.8096741 & 5 & 7 & 2 \\
\hline c...(... & 3.9992170 & 4.0612326 & 4.4978479 & 4 & 10 & 3 \\
\hline c...(...c... & 1.7523875 & 2.5921235 & 0.9359654 & 24 & 19 & 13 \\
\hline c...(...Br.. & 1.3392341 & 0.5340779 & 1.1889034 & 17 & 0 & 0 \\
\hline с...(... ... & 1.0002010 & 0.2472155 & -0.2478234 & 19 & 41 & 13 \\
\hline с...(...Cl.. & 1.1825597 & 2.5039425 & 0.9088978 & 15 & 23 & 7 \\
\hline с...(... & 1.1553993 & 0.9107402 & 1.8401083 & 10 & 34 & 6 \\
\hline c...(...N... & -0.4647652 & -0.5109250 & 0.5049629 & 13 & 41 & 11 \\
\hline 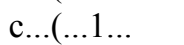 & 3.2546395 & 1.6826438 & 1.7521385 & 17 & 17 & 10 \\
\hline c...(...O $=.$. & 2.0008786 & 2.9044603 & 2.8172049 & 15 & 17 & 7 \\
\hline c...(..F... & 2.5615727 & 2.2483847 & 2.9956341 & 6 & 0 & 2 \\
\hline с...1...О... & 0.3157218 & 0.2460029 & 0.0026575 & 7 & 3 & 3 \\
\hline c...1...С. $\ldots$ & -0.3426326 & -0.4053179 & -0.0587013 & 10 & 10 & 4 \\
\hline c...1...... & 4.1291472 & 3.7385617 & 4.5010102 & 15 & 17 & 6 \\
\hline c...1...c... & 2.5270201 & 4.1254591 & 1.6392878 & 64 & 69 & 24 \\
\hline с...2...c... & 3.1834674 & 3.5765233 & 2.5649848 & 46 & 41 & 10 \\
\hline с $\ldots 2 \ldots \mathrm{O} \ldots$ & -2.1902681 & -0.8169002 & -1.5600959 & 6 & 5 & 0 \\
\hline с ...2...С ... & 2.0599980 & 3.3166075 & 2.0508240 & 6 & 4 & 2 \\
\hline c...2...... & -3.4837706 & -2.7454921 & -2.2497303 & 5 & 2 & 2 \\
\hline с $\ldots 3 \ldots$.... & 0.5671953 & 0.3280375 & 2.5577465 & 14 & 15 & 6 \\
\hline с ...C ...C ... & -0.4968006 & -1.0630716 & -0.7464899 & 4 & 6 & 0 \\
\hline с...N...(... & 4.1212930 & 5.1280879 & 3.2342653 & 9 & 3 & 3 \\
\hline с...О...(.. & 7.8795041 & 8.7529500 & 8.5448576 & 5 & 2 & 0 \\
\hline с...О...С ... & 0.4969760 & 1.0602889 & 1.0035994 & 10 & 8 & 0 \\
\hline с...с...2... & -1.0046754 & -0.7477295 & -1.3148992 & 59 & 58 & 20 \\
\hline c...c...c... & -0.9189229 & -1.1362229 & -0.9886103 & 171 & 148 & 50 \\
\hline с...c...1... & 0.9684404 & 1.0961687 & 1.0267859 & 111 & 101 & 36 \\
\hline с...c...3... & -1.4056269 & -2.8661586 & -1.7490548 & 18 & 24 & 6 \\
\hline с...с...4 ... & -1.2498300 & 0.6278968 & 0.4997288 & 5 & 5 & 4 \\
\hline c...c...(... & -0.5592802 & -0.7452834 & -0.2831183 & 87 & 110 & 45 \\
\hline с ...n...1... & 0.4037274 & 0.7545182 & 1.8635708 & 8 & 9 & 8 \\
\hline $\mathrm{n} \ldots 1 \ldots \mathrm{c} \ldots$ & 1.1446162 & 1.1906216 & 0.1368885 & 11 & 8 & 8 \\
\hline n...c...c... & -4.4951810 & -4.4955509 & -4.2500468 & 5 & 11 & 1 \\
\hline n...c....... & -1.7475062 & -0.9098866 & -0.0016730 & 10 & 13 & 13 \\
\hline о...(....... & 1.9983265 & -0.3077248 & 1.1610603 & 5 & 5 & 5 \\
\hline o...1...... & -0.8795536 & -0.8151611 & -1.4961309 & 4 & 3 & 2 \\
\hline s...1...... & 3.0007359 & 3.3126224 & 2.8719278 & 5 & 5 & 6 \\
\hline
\end{tabular}


Figure 5. Graphical representation of the model for $\log \mathrm{TD}_{50}$ calculated with Equation 3.

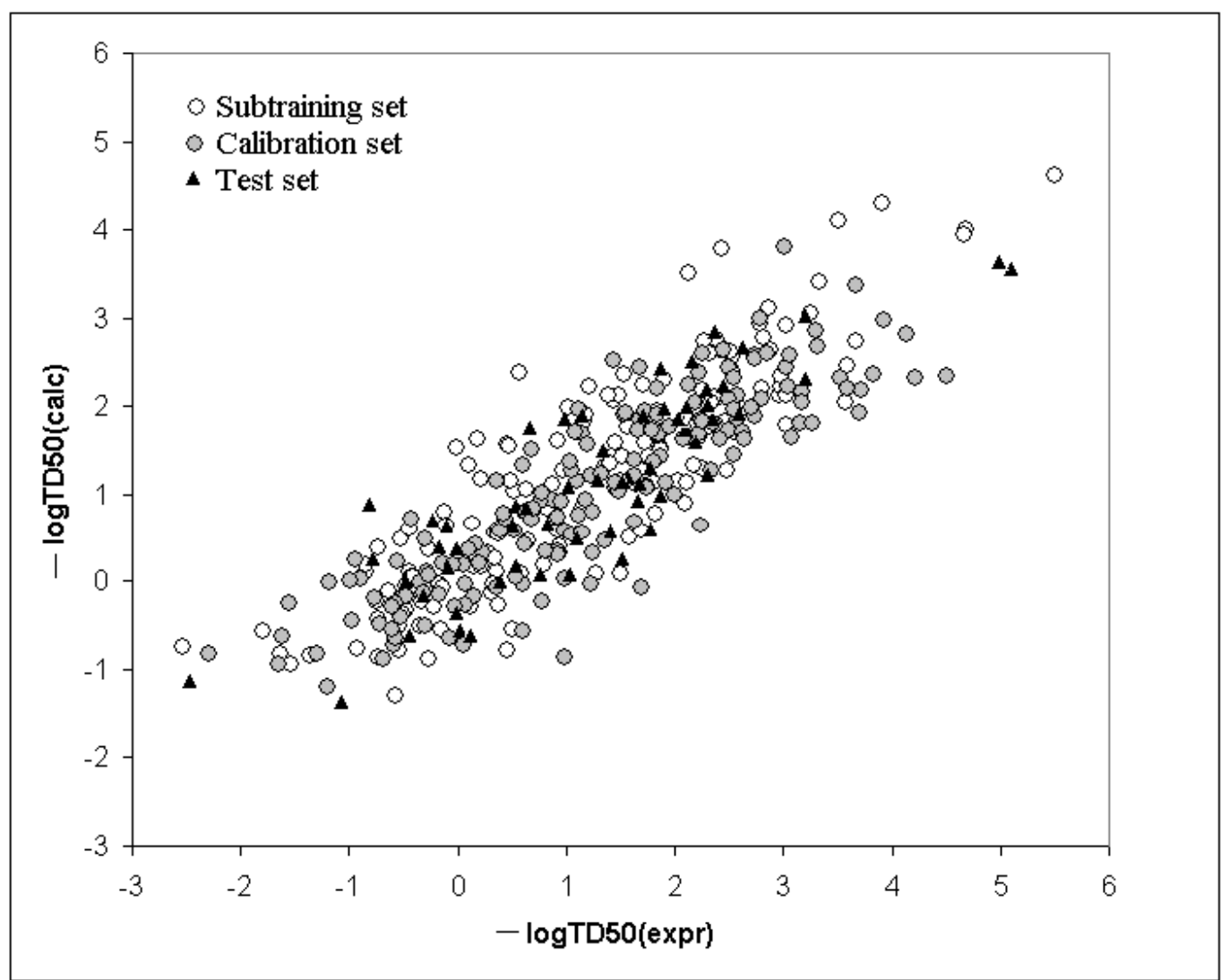

\section{Discussion}

One can see that the statistical characteristics of this model are reasonably good. As additional validation we have calculated Y-scrambling criterion, randomly shifting the carcinogenicity values [16,17]. If after the shifting (300 exchanges recommended in Ref.[17]) the correlation coefficient is less than 0.2 , the correlation of our model can be classified as not chance correlation. Thus, the Y-scrambling has shown that the Equation 3 gives robust prediction (not chance correlation) for the test set.

In our previous study we examined different equations for the carcinogenicity model, and only one split into the subtraining, calibration and test set [15]. Examination of three splits indicates that good results occur for all three splits (Table 4). Thus, we expect that the present model is more robust, also considering the Y-scrambling test.

One can see from Table 5 that there are three categories of SMILES attributes: category 1 is the set of SMILES attributes with the correlation weight more than zero in all three probes of the Monte Carlo optimization; category 2 is the set of SMILES attributes with the correlation weight less than zero in all three probes; category 3 is the set of SMILES attributes with non consistent values, which have both correlation weights more than zero and correlation weights less zero in the three probes of the optimization. We can say that the category 1 contains promoters of $\log \mathrm{TD}_{50}$ increase; category 2 contains promoters of $\log \mathrm{TD}_{50}$ decrease; category 3 contains attributes with unclear influence on $\log \mathrm{TD}_{50}$. 
The !-02, \#, Cl, S, [N+], and [O-] SMILES elements are promoters of logTD50 increase, thus of carcinogenicity. However it is necessary to take into account the value of correlation weight as well as the number of the given attribute in the subtraining set. Taking this into account, one can detect that the strongest promoters of the $\log \mathrm{TD} 50$ increase are $\mathrm{Cl}$ (number $\mathrm{Cl}$ in the subtraining set is 61, the range of correlation weights of the $\mathrm{Cl}$ in three probes is 2.19 - 3.19) and [O-] (the number of [O-] in the subtraining set is 26 , the range of correlation weights in three probes is $5.92-6.96$ ).

A similar analysis can be done for the promoters of $\log \mathrm{TD}_{50}$ decrease. For instance, the number of bracket s' (' in the subtraining set is 708 and the range of correlation weights of bracket is from -1.366 till -1.686; the number of ' $=$ ' ' in the subtraining set is 77 and the range of correlation weight is from 1.866 till 2.144. Table 6 contains examples of compounds, which contain the mentioned SMILES attributes. Thus, the analysis of the correlation weights of SMILES attributes can help in searching for agents of the carcinogenicity phenomenon.

An important feature of our model is that SMILES attributes are used for the QSAR predicted values and not only as tool for a binary classification (carcinogenic or not). Our model, which provides continuous values, can be used for risk assessment calculations, where a dose is necessary.

Table 6. Examples of compounds which contain promoters of increase/decrease of the $\log \mathrm{TD}_{50}$.

\begin{tabular}{|l|l|l|}
\hline Structure & CAS and SMILES & log TD \\
\hline
\end{tabular}

*) One can see that aromatic bonds are indicated in SMILES by 'c' (lower case), thus '=' is indicator of local double bonds which are not a part of aromatic fragments. 
The applicability domain for these models can be defined from a probabilistic point of view: one can estimate the carcinogenic potential of compound if the SMILES of this compound does not contain rare SMILES attributes. A stronger definition of the applicability domain can be formulated taking into account the roles of the attributes (as promoters of $\log \mathrm{TD}_{50}$ increase/decrease): thus, one can estimate the carcinogenic potential of a compound if the SMILES of the compound contains solely apparent promoters of $\log \mathrm{TD}_{50}$ increase and/or decrease (without of SMILES attributes with unclear role).

\section{Conclusions}

- Optimal descriptors calculated by the Monte Carlo method can provide reasonable prediction for the carcinogenicity $\log (\mathrm{TD} 50)$.

- Blocking of rare SMILES attributes can improve statistical quality of the predicting. Splits into subtraining, calibration and test sets, as well splits into the training and test sets have influence to statistical characteristics of the models. In our case, in three splits examined in this study these characteristics are similar.

- $\quad$ The correlation balance, i.e., the [Subtraining-Calibration-Test] system gave models which are better in comparison with models obtained with the more traditional [Training-Test] system.

\section{Acknowledgements}

The authors thank the Marie Curie Fellowship (the contract ID 39036, CHEMPREDICT) and the EC project CAESAR (Project no. 022674 (SSPI)) for financial support.

\section{References and Notes}

1. Benfenati, E.; Benigni, R.; Demarini, D.M.; Helma, C.; Kirkland, D.; Martin, T.M.; Mazzatorta, P.; Ouedraogo-Arras, G.; Richard, A.M.; Schilter, B.; Schoonen, W.G.; Snyder, R.D.; Yang, C. Predictive Models for Carcinogenicity and Mutagenicity: Frameworks, State-of-the-Art, and Perspectives. J. Environ. Sci. Health C Environ. Carcinog. Ecotoxicol. Rev. 2009, 27, 57-90.

2. Benigni, R.; Netzeva, T.; Benfenati, E.; Bossa, C.; Franke, R.; Helma, C.; Hulzebos, E.; Marchant, C.; Richard, A.; Woo, Y.-T.; Yang, C. The expanding role of predictive toxicology: An update on the (Q)SAR models for mutagens and carcinogens. J. Environ. Sci. Health C 2007, 25, 53-97.

3. Benigni, R. Structure-activity relationship studies of chemical mutagens and carcinogens: Mechanistic investigations and prediction approaches. Chem. Rev. 2005, 105, 1767-1800.

4. Contrera, J.F.; MacLaughlin, P.; Hall, L.H.; Kier, L.B. QSAR modeling of carcinogenic risk using discriminant analysis and topological molecular descriptors. Curr. Drug Dis. Technol. 2005, 2, 55-67.

5. Weininger, D. SMILES, a chemical language and information system. 1. Introduction to methodology and encoding rules. J. Chem. Inf. Comput. Sci. 1988, 28, 31-36. 
6. Weininger, D.; Weininger, A.; Weininger, J.L. SMILES. 2. Algorithm for generation of unique SMILES notation. J. Chem. Inf. Comput. Sci. 1989, 29, 97-101.

7. Weininger, D. SMILES. 3. DEPICT. Graphical depiction of chemical structures. J. Chem. Inf. Comput. Sci. 1990, 30, 237-243.

8. Vidal, D.; Thormann, M.; Pons, M. LINGO, an efficient holographic text based method to calculate biophysical properties and intermolecular similarities. J. Chem. Inf. Model. 2005, 45, 386-393.

9. Toropov, A.A.; Benfenati, E. Optimisation of correlation weights of SMILES invariants for modelling oral quail toxicity. Eur. J. Med. Chem. 2007, 42, 606-613.

10. Toropov, A.A.; Benfenati, E. Additive SMILES-based optimal descriptors in QSAR modelling bee toxicity: Using rare SMILES attributes to define the applicability domain. Bioorg. Med. Chem. 2008, 16, 4801-4809.

11. Toropov, A.A.; Rasulev, B.F.; Leszczynski, J. QSAR modeling of acute toxicity by balance of correlations. Bioorg. Med. Chem. 2008, 16, 5999-6008.

12. Toropov, A.A.; Toropova, A.P. QSAR Modeling of Mutagenicity Based on Graphs of Atomic Orbitals Internet Electron. J. Mol. Des. 2002, 1, 108-114.

13. Marino, D.J.G.; Peruzzo, P.J.; Castro, E.A.; Toropov, A.A. QSAR Carcinogenic Study of Methylated Polycyclic Aromatic Hydrocarbons Based on Topological Descriptors Derived from Distance Matrices and Correlation Weights of Local Graph Invariants Internet Electron. J. Mol. Des. 2002, 1, 115-133.

14. Peruzzo, P.J.; Marino, D.J.G.; Castro, E.A.; Toropov, A.A. QSPR Modeling of Lipophilicity by Means of Correlation Weights of Local Graph Invariants Internet Electron. J. Mol. Des. 2003, 2, 334-347.

15. Available online: http://chem.sis.nlm.nih.gov/chemidplus/.

16. Available online: http://webbook.nist.gov/chemistry/.

17. Available online: http://www.epa.gov/ncct/dsstox/sdf_cpdbas.html/.

18. Toropov, A.A.; Toropova, A.P.; Benfenati, E.; Manganaro, A. QSAR modelling of carcinogenicity by balance of correlations. Mol. Divers. 2009, in press.

19. Mazzatorta, P.; Smiesko, M.; Piparo, E.; Benfenati, E. QSAR model for predicting pesticide aquatic toxicity. J. Chem. Inf. Model. 2005, 45, 1767-1774.

20. Fatemi, M.H.; Haghdadi, M. Quantitative structure-property relationship prediction of permeability coefficients for some organic compounds through polyethylene membrane. J. Mol. Struct. 2008, 886, 43-50.

(C) 2009 by the authors; licensee Molecular Diversity Preservation International, Basel, Switzerland. This article is an open-access article distributed under the terms and conditions of the Creative Commons Attribution license (http://creativecommons.org/licenses/by/3.0/). 